\title{
Energetics and mixing of stratified, rotating flow over abyssal hills
}

By Varvara E. Zemskova (barbara.zemskova@utoronto.ca) and

Nicolas Grisouard (nicolas.grisouard @ utoronto.ca)

University of Toronto, Department of Physics, 60 St. George Street, Toronto ON M5S 1A7, Canada

This paper is a non-peer reviewed preprint submitted to EarthArXiv. It was submitted to Journal of Physical Oceanography on 9 July, 2021 and is currently undergoing peer review.

Please feel free to reach out to us if you have any questions or comments regarding the paper. 


\title{
Energetics and mixing of stratified, rotating flow over abyssal hills
}

\author{
Varvara E. Zemskova* and Nicolas Grisouard
}

Department of Physics, University of Toronto, Toronto, ON, Canada

${ }_{4}{ }^{*}$ Corresponding author: Varvara E. Zemskova, barbara.zemskova@ utoronto.ca 
ABSTRACT

${ }_{5}$ One of the proposed mechanisms for energy loss in the ocean is through dissipation of internal 6 waves, in particular above rough topography where internal lee waves are generated. Rates of 7 dissipation and diapycnal mixing are often estimated using linear theory and a constant value for mixing efficiency. However, previous oceanographic measurements found that non-linear dynamics may be important close to topography. In order to investigate the role of non-linear interactions, we conduct idealized 3D numerical simulations of steady flow over 1D topography and vary the topographic height, which correlates to the degree of flow non-linearity. We analyze spatial distribution of energy transfer rates between internal waves and the non-geostrophic portion of time-mean flow, and of dissipation and diapycnal mixing rates. In our simulations with taller, more non-linear topographies, energy transfer rates are similar to previously unexplained oceanographic observations near topography: internal waves gain energy from time-mean flow through horizontal straining and lose energy through vertical shearing. In the tall topography simulations, buoyancy fluxes also play a significant role, consistent with observations but contrary to linear wave theory, suggesting that quasigeostrophy-based approximations and linear theory may not hold in some regions above rough topography. Both dissipation and mixing rates increase with topographic height, but their vertical distributions differ between topographic regimes. As such, vertical profile of mixing efficiency is different for linear and non-linear topographic regimes, which may need to be incorporated into parameterizations of small-scale processes in models and estimates of ocean energy loss. 


\section{Introduction}

Energy input into the ocean from wind work, differential surface buoyancy forcing, and tides is eventually lost through small-scale turbulent processes (Munk and Wunsch 1998; Wunsch and Ferrari 2004; Hughes et al. 2009; Zemskova et al. 2015). One of the important pathways to dissipation is the breaking of internal waves generated as a result of interactions between a steady geostrophic (e.g., Scott et al. 2011; Nikurashin and Ferrari 2010a; Waterman et al. 2013), eddy (e.g., Liang and Thurnherr 2012; Whalen et al. 2018; Yang et al. 2018), or tidal flow (e.g. Legg and Klymak 2008; Musgrave et al. 2017) with bottom topography. Dissipation and mixing rates have a high degree of spatial and temporal variability (De Lavergne et al. 2016; Mashayek et al. 2017b), and in-situ measurements have been taken primarily in the known hot spots, such as the Southern Ocean and in particular the Drake Passage (Sheen et al. 2013; Waterman et al. 2014; Brearley et al. 2013), over the Hawaiian Ridge (Klymak et al. 2008; Sun and Pinkel 2012), or in the South China Sea (Alford et al. 2015; Hu et al. 2020). These regions are generation sites for topographic lee waves, whose breaking enhances both kinetic energy dissipation and irreversible mixing rates (Legg 2021).

Spatial resolution of global and regional ocean models is often insufficient to capture small topographic features, and internal motions generated via flow-topography interactions have to be parameterized. For lee waves, many such parameterizations rely on linear theory of a steady, homogeneous flow with velocity $U$ and buoyancy frequency $N$ passing over a sinusoidal topographic obstacle with wavenumber $k$ and height $h_{0}$ (Bell 1975). Given the dispersion relation of internal waves, lee waves freely propagate away from the topography if

$$
\chi=\frac{U k}{N} \in\left(\frac{|f|}{N}, 1\right),
$$


where $f$ is the local Coriolis parameter. Linear theory then estimates the amount of energy converted from the steady background flow into the lee wave field, given an approximate bottom topography spectrum and near-bottom velocity and stratification values (Nikurashin and Ferrari 2010b).

Linear theory prediction for energy flux into the lee waves has been commonly used to estimate dissipation and mixing rates above rough topography (e.g., Nikurashin and Vallis 2011; Nikurashin and Ferrari 2013; Waterhouse et al. 2014; De Lavergne et al. 2016). This parameterization assumes that a certain fraction of the energy is dissipated locally and that dissipation has a particular vertical profile, though the details of these assumptions may vary across studies (for a thorough review, see Legg 2021). Furthermore, dissipation rates are related to diapycnal diffusivity through flux coefficient, defined as

$$
\Gamma=\frac{\eta}{1-\eta},
$$

where the mixing efficiency $\eta$ is the ratio of energy lost to irreversible mixing to the total mechanical energy loss, i.e., the sum of dissipation and irreversible mixing (Peltier and Caulfield 2003).

In studies of lee waves and bottom topography-driven flows, the canonical value of $\Gamma=0.2$ has been commonly used (Mashayek et al. 2017b). Yet, numerous other studies have shown that the value for $\Gamma$ varies depending on physical processes and geostrophic environments (see details in the review by Caulfield 2021). Global models, and especially climate models, rely on an accurate parameterization of diapycnal diffusivity to compute the strength of the meridional overturning circulation, which plays an important role in the uptake and distribution of heat and nutrients in the ocean. However, because of the limitations of previous numerical and observational studies, there is a paucity of estimates for $\Gamma$ specifically for bottom-driven flows. In this study, one of our goals is to provide such estimates through a series of idealized numerical simulations and illustrate that mixing efficiency, and subsequently, $\Gamma$ can be indeed spatially variable. 
Linear theory also relies on the wave steepness parameter, or inverse Froude number, defined as

$$
J=\frac{N h_{0}}{N}
$$

being small. For a supercritical value $J>1$, linear theory no longer holds, and non-linear processes become important (Klymak et al. 2010; Winters and Armi 2012). While linear and non-linear topographic regimes form a continuous spectrum, for brevity, we will refer to topographies with $J<1$ as linear and with $J>1$ as non-linear. Recently, numerical simulations by Zemskova and Grisouard (2021) showed that non-linear resonant interactions between lee waves and nearinertial waves, which are both generated in the case of bottom-driven flows over lee-wave radiative topography, are particularly strong for non-linear topographies and enhance dissipation rates both near and away from the topography.

The importance of non-linear topography has also been seen in the observations by Cusack et al. (2020). They found energy transfer rates between the time-mean flow and the internal waves near the bottom in the Southern Ocean that were inconsistent with the linear internal wave theory predictions, but were unable to identify specific mechanisms. Estimates of these energy transfer rates have been used to parameterize turbulent viscosity coefficients (Ruddick and Joyce 1979; Brown and Owens 1981; Polzin 2009) and estimate the energy loss of wind work in the ocean interior (Ferrari and Wunsch 2009). However, in the Eulerian framework, lee waves have zero frequency, they may appear in the time-mean component, and their interactions with other internal waves may be captured in the energy transfer rates computed from the observations. Our second goal in this study is to illuminate how deviations from quasigeostrophy (QG) and linearity in the bottom-driven flows affect these energy exchange rates. Indeed, we will show that over non-linear topographies, the observations of Cusack et al. (2020) can be qualitatively reproduced, in part because of the interaction between lee waves and near-inertial waves. 
With these two goals in mind, namely, diagnosing spatial patterns of mixing efficiency and exploring deviations from common approximations, we focus on the lee-wave radiative topography, but vary the topographic height in our idealized numerical simulations in order to compare the linear and non-linear regimes. This paper builds on the findings of Zemskova and Grisouard (2021), and we briefly describe the set-up of our simulations in $\$ 2$. We then summarize our energetics framework in $\S 3$ by separating the kinetic energy (KE) and available potential energy (APE) reservoirs based on the temporal frequency spectra in $§ 3 a$ and defining the relevant energy transfer and dissipation rates in $\S 3 \mathrm{~b}-\mathrm{c}$, which we compare with the QG theory assumptions in $\S 3 \mathrm{~d}$. In $\S 4$, we compare KE and APE exchange rates between the time-mean flow to internal waves across the topographic heights and find that the energy exchange rates between lee waves and internal waves over non-linear topography, but not linear topography, are consistent with the ocean observations. Furthermore, in $\S 5$, we explore the effects of topographic height on KE and APE dissipation rates and mixing efficiency, and find that the vertical distribution of mixing efficiency varies significantly between the linear and non-linear regimes. Finally, in $§ 6$, we relate our findings back to the ocean, emphasizing that non-linear topographies may be important and need to be considered in parameterizations of turbulent viscosity and diffusivity coefficients used in ocean models.

\section{Model set-up}

The details and the justification of the set-up for the numerical simulations are described in Zemskova and Grisouard (2021). Here, we summarize the main features of the idealized rectangular domain shown in Fig. 1. 
We solve the nonhydrostatic rotating Navier-Stokes equations in the Boussinesq approximation, namely

$$
\begin{array}{r}
\frac{\partial \hat{\mathbf{u}}}{\partial \hat{t}}+\hat{\mathbf{u}} \cdot \nabla \hat{\mathbf{u}}+\hat{f} \mathbf{k} \times \hat{\mathbf{u}}=-\frac{\nabla \hat{p}}{\rho_{0}}+\hat{b} \mathbf{k}+\hat{v} \nabla^{2} \hat{\mathbf{u}}+\hat{f} \hat{U} \mathbf{j} \\
\frac{\partial \hat{b}}{\partial \hat{t}}+\hat{\mathbf{u}} \cdot \nabla \hat{b}=\hat{\kappa} \nabla^{2} \hat{b} \quad \text { and } \quad \nabla \cdot \hat{\mathbf{u}}=0
\end{array}
$$

using Nek5000, a spectral-element code (Fischer et al. 2008). Here, $\hat{\mathbf{u}}=(\hat{u}, \hat{v}, \hat{w})$ is velocity in Cartesian across-ridge, along-ridge and upward vertical directions $(\mathbf{i}, \mathbf{j}, \mathbf{k})$, respectively. These directions are associated with coordinates $(\hat{x}, \hat{y}, \hat{z})$, respectively, $\hat{b}=-\hat{g}\left(\hat{\rho}-\hat{\rho}_{0}\right) / \hat{\rho}_{0}$ is buoyancy, with $\hat{\rho}$ the density and $\hat{\rho}_{0}$ a constant reference density, $\hat{p}$ is pressure, $\hat{U}$ is a constant, crossridge geostrophic velocity we prescribe, $\hat{v}$ is kinematic viscosity, and $\hat{\kappa}$ is diffusivity ( $\hat{\circ}$ represent dimensional quantities). The body force $\hat{f} \hat{U}$ applied to the $y$-momentum equation represents a barotropic pressure gradient that geostrophically balances a mean flow at all depths (Nikurashin and Ferrari 2010b; Klymak 2018). All physical variables are non-dimensionalized using ocean depth $\hat{H}$ for length scales, $1 / \hat{f}$ for time scales, and buoyancy $\hat{H} \hat{N}^{2}$ for buoyancy. The DNS formulation of the Navier-Stokes equations resolves fluid motions from the domain scales to the smallest spatio-temporal dissipative scales without employing subgrid turbulence parametrization or filtering. As such, we are able to compute all terms of the KE and APE budgets, including dissipation and irreversible mixing, directly.

The domain is doubly-periodic in the $x$ and $y$ directions with a no-slip bottom boundary, with a bottom height defined by

$$
h(x)=h_{0} \sin ^{2}(k x / 2),
$$

where $h_{0}$ is the maximum topographic height. The domain size is $L_{x}=L_{y}=2 \pi / k$ and $H=2 L_{x}$. The top surface is a rigid lid with no-buoyancy-flux and no-slip boundary conditions. We restrict our analysis to above the bottom boundary layer to exclude effects of the no-slip boundary and below 
$H A B=0.5$ ( $H A B$ is height above the bottom), to exclude any downward-propagating reflections from the top boundary. We set Prandtl number $\operatorname{Pr}=v / \kappa=1$, where $v$ is kinematic viscosity, $\kappa$ is buoyancy diffusivity.

In this study, we focus on the effects of hydraulic control and increased non-linearity for leewave radiating topography, for which linear theory (e.g. Bell 1975; Müller 1976; Ruddick and Joyce 1979 ) is often applied to estimate the energy budget terms. For all experiments, we fix $f / N=0.1$ so that the radiative (lee wave) regime corresponds to $\chi=U k / N \in[0.1,1]$, and choose $\chi=0.16$. We conduct four numerical experiments with the inverse Froude number $J=N h_{0} / U=(0.6,1,2)$ by varying the topographic height $h_{0}$. This range of $J$ is chosen to capture the transition from linear to non-linear regime, in contrast to the highly non-linear regimes $(J=O(10-100))$, investigated in other previous studies of stratified flows over topography (e.g. Klymak et al. 2010; Jagannathan et al. 2020). We initialize the simulations with $U$ and $B(z)=N^{2} z$. We run the simulations with $J=(2,5)$ until $t=50$, corresponding to approximately $8 t_{I}$, with $t_{I}=2 \pi / f$ the inertial period. We run the simulation with $J=0.6$ until $t=12 t_{I}$ to allow for at least six breaking events, which occur every $t_{I}$, as shown in the Hovmöller diagram of the KE dissipation in Figure 2. This periodic breaking plays an important role in dissipation rates in the interior, as described in Zemskova and Grisouard (2021). For the following analysis in this paper, we consider a temporal average over the last four inertial periods.

\section{Energetics framework}

\section{a. Energy reservoirs}

We separate the velocity, $(u+U, v, w)$, and buoyancy, $b$, fields into four components: geostrophic flow, ageostrophic time-mean component $\left({ }^{\circ}\right)$, internal wave $\left({ }^{\prime}\right)$, and high-frequency $(\cdot H F)$ compo- 
nents, i.e.,

$$
\begin{array}{r}
\mathbf{u}(x, y, z, t)=U \mathbf{i}+\overline{\mathbf{u}}(x, y, z)+\mathbf{u}^{\prime}(x, y, z, t)+\mathbf{u}_{H F}(x, y, z, t) \quad \text { and } \\
b(x, y, z, t)=B(z)+\bar{b}(x, y, z)+b^{\prime}(x, y, z, t)+b_{H F}(x, y, z, t) .
\end{array}
$$

What we refer to as ageostrophic time-mean (hereafter shortened into time-mean) fields $(\overline{\mathbf{u}}, \bar{b})$ here include all zero-frequency flows and most notably the lee waves, which have zero-frequency in the topographic reference frame, but exclude the geostrophic flow.

We find a noticeable time-scale separation between the near-zero frequency $(\omega \approx 0)$ motions and motions at frequencies greater than $f$ as shown by KE and APE (defined below) spectra in Fig. 4, which allows us to consider the zero-frequency and internal wave reservoirs separately. While internal waves by definition have frequencies in the $[f, N]$ band, because the inertial peak is somewhat broad, in order to account for energy shift in our idealized set-up, we define near-inertial motions to have frequencies between $0.75 f$ and $1.25 f$ included (the values are constrained by the temporal resolution of our output). The super-inertial internal waves are then defined as motions with frequencies between $1.5 f$ and $N$ included. Although the issue of Doppler shifting may necessitate Lagrangian filtering of the energy equations (cf. e.g., Shakespeare and Hogg 2017), our analysis is conducted in the Eulerian framework in order to compare our results with the analysis of observational measurements (e.g., Polzin 2010; Sun and Pinkel 2012; Cusack et al. 2020), which are also reported in the Eulerian reference frame. In the Eulerian framework, stationary or quasi-stationary lee waves have zero-frequency, and thus may be included in the time-mean component. We specifically separate the time-mean component, which includes the lee waves in our simulations, from the geostrophic flow to test the assumptions of the QG limit.

In this study, we focus on the time-mean (i.e., zero-frequency minus geostrophic flow) and internal wave (i.e., $\omega \in[0.75 f, N]$ frequency band) motions. Although internal waves also include lee waves, throughout the text we will refer to motions with frequencies $\omega \in[0.75 f, N]$ as simply 
internal waves for brevity, as lee waves are a part of the time-mean flow. The total energy in each component is the sum of kinetic (KE) and available potential energy (APE) reservoirs. We define kinetic energy $E_{K}$, divided by $\rho_{0}$, for the time-mean and internal wave components, respectively, $E_{K}^{m}$ and $E_{K}^{i w}$, as

$$
\begin{gathered}
E_{K}^{m}=\frac{1}{2}\left(\bar{u}^{2}+\bar{v}^{2}+\bar{w}^{2}\right) \quad \text { and } \\
E_{K}^{i w}=\frac{1}{2}\left(\overline{u^{\prime} u^{\prime}}+\overline{v^{\prime} v^{\prime}}+\overline{w^{\prime} w^{\prime}}\right) .
\end{gathered}
$$

The APE, $E_{A}$, is the difference between the total gravitational potential energy and the background potential energy, $E_{B}=-b z^{*}$, which is the minimum potential energy of the system if all water parcels were resorted adiabatically according to their densities (Winters et al. 1995). We find the reference background buoyancy of a water parcel, $b^{*}(z)$, by resorting fluid parcels in a monotonicallyincreasing order of density with depth. The corresponding height of each parcel in the background state is $z^{*}(b)$, which varies both spatially and temporally, as it and $E_{B}$ both depend on the resorting of the instantaneous $b(x, y, z, t)$ field. Following previous works (Scotti and White 2014; Zemskova et al. 2015, 2020), we define the total APE locally as the sum of the time-mean and turbulent components, such that

$$
\begin{aligned}
E_{A} & =\int_{b^{*}(z, t)}^{b(\mathbf{x}, t)}\left[z-z^{*}(s, t)\right] d s \\
& =E_{A}^{m}+E_{A}^{t}=\int_{\bar{b}^{*}(z)}^{\bar{b}(\mathbf{x})}\left[z-z^{*}(s)\right] d s+E_{A}^{t} .
\end{aligned}
$$

Here, $b^{*}$ is the buoyancy of the resorted buoyancy field at a given height such that $z^{*}\left(b^{*}\right)=z$. All potential energy from the geostrophic flow is in the background potential energy reservoir because $B=N^{2} z$, with $N^{2}$ a positive constant. Because $z^{*}$ is non-linear such that $\left[z^{*}\left(b_{1}\right)+z^{*}\left(b_{2}\right)\right] / 2 \neq$ $z^{*}\left(\left(b_{1}+b_{2}\right) / 2\right)$, we do not separate the turbulent component $E_{A}^{t}=E_{A}-E_{A}^{m}$ into the internal waves and higher frequency waves. However, rather than the total energy in the reservoirs, we are 
interested in the energy exchange rates between the reservoirs as well as the dissipation rates, which can be computed for the $[f, N]$ frequency band separately as described in the next subsections.

\section{b. Complex demodulation}

Complex demodulation (CD) filtering has been previously applied to the internal wave fields to isolate motions at a given frequency (Mercier et al. 2008; Grisouard and Thomas 2015). The specifics of our techniques are described in detail in Zemskova and Grisouard (2021), which we briefly summarize below.

Here, we focus on the near-inertial waves in particular because they have been demonstrated to play an important role in the dynamics of bottom-driven flows (e.g. Nikurashin and Ferrari 2010a; Zemskova and Grisouard 2021). From the internal wave field, we can isolate motions at any given frequency $n f$ by computing

$$
\tilde{q}_{n f}=\frac{1}{T} \int_{t_{0}}^{t_{0}+T} q(x, z) e^{-i n f t} d t
$$

where $q=(u, v, w, b)$. In this study, we specifically consider inertial motions, i.e., $\tilde{q}_{f}$. Initial time $t_{0}$ is large enough for the dynamics to have become reasonably stationary, and which we take as $t_{0}=4 t_{I}$ for $J=1,2$ and as $t_{0}=8 t_{I}$ for $J=0.6$, and choose $T=4 t_{I}$, multiple of $2 \pi / f$.

We also compute the non-linear terms that force motions at $\omega=f$, namely:

$$
\Lambda_{f}=\frac{1}{T} \int_{t_{0}}^{t_{0}+T}-\left(u u_{x}+w u_{z}\right) e^{-i f t} d t .
$$

$\Lambda_{f}$ represents the sum of the triadic non-linear interactions between the inertial signal $(\omega=f)$ and all other frequency pairs $\left(\omega_{1}, \omega_{2}\right)$ such that $\omega_{1}+\omega_{2}=f$. If $\Lambda_{f}>0(<0)$, then nonlinear interactions transfer energy to (away from) the inertial motions. 


\section{c. Energy budget terms}

Here, we specifically emulate the energy term calculations presented in Cusack et al. (2020). We focus on the energy sinks (KE dissipation and irreversible mixing) and exchange between the time-mean field and internal waves, and the analysis of the full KE and APE budgets are beyond the scope of this study. The energy budget terms considered in this paper are summarized in a diagram in Figure 3. The internal wave components can be computed by integrating the cospectrum (real part of the cross spectrum), $C(\omega)$, for example, over $[f, N]$ frequencies as in Cusack et al. (2020). Namely, for any two internal wave quantities $\alpha^{\prime}$ and $\beta^{\prime}$, the temporally-averaged cross correlation terms are computed as

$$
\overline{\alpha^{\prime} \beta^{\prime}}=\int_{f}^{N} C_{\alpha \beta}(\omega) d \omega .
$$

We carry out the frequency analysis (i.e., computing spectra and cospectra) at each $(x, y, z)$ over the last $4 t_{I}$ of each simulation prior to any horizontal averaging in order to capture all waves. Analogous to Eqn. (11), we compute the energy budget terms at near-inertial frequencies by integrating over $\omega \in[0.75 f, 1.25 f]$ and denote these energy budget terms with subscript or superscript $f$. We also compute the energy budget terms for super-inertial internal waves by integrating over $\omega \in[1.5 f, N]$ and denote these energy budget terms with subscript or superscript SIW (for super-inertial waves). To be precise, we define energy transfers to-and-from near-inertial waves and to-and-from superinertial waves, respectively, as

$$
\begin{aligned}
\overline{\alpha^{\prime} \beta^{\prime}} & =\int_{0.75 f}^{1.25 f} C_{\alpha \beta}(\omega) d \omega, \quad \text { and } \\
\overline{\alpha^{\prime} \beta^{\prime}}{ }_{S I W} & =\int_{1.5 f}^{N} C_{\alpha \beta}(\omega) d \omega .
\end{aligned}
$$

Following a mean-eddy decomposition, where an "eddy" field is taken to be deviation from the time-averaged flow, of KE and APE energy budget equations (e.g., Scotti and White 2014; Shakespeare and Hogg 2017), and applying Eqn. (11) to the internal wave terms, we define time- 
averaged KE dissipation rates for the time-mean component and internal waves, respectively, as

$$
\begin{array}{r}
D\left(E_{K}^{m}\right)=v|\nabla \overline{\mathbf{u}}|^{2} \quad \text { and } \\
D\left(E_{K}^{i w}\right)=v \overline{\left|\nabla \mathbf{u}^{\prime}\right|^{2}}=v \int_{\omega_{1}}^{\omega_{2}} C_{|\nabla \mathbf{u}|^{2}}(\omega) d \omega .
\end{array}
$$

Time-averaged irreversible mixing rates, which are the APE dissipation rates due to diabatic fluxes (Winters and D'Asaro 1996) for the time-mean component and internal waves are defined as

$$
\begin{aligned}
& D\left(E_{A}^{m}\right)=\left.\kappa|\nabla \bar{b}|^{2} \frac{d z^{*}}{d b}\right|_{b=\bar{b}} \quad \text { and } \\
& D\left(E_{A}^{i w}\right)=\kappa\left|\nabla b^{\prime}\right|^{2} \frac{d z^{*}}{d b}=\kappa \int_{\omega_{1}}^{\omega_{2}} C_{|\nabla b|^{2} \frac{d z^{*}}{d b}}(\omega) d \omega,
\end{aligned}
$$

where we recall that in our simulations, $\kappa=v$. In Eqns. (13) and (14), we bound the integrals with $\omega_{1}=0.75 f, \omega_{2}=1.25 f$ for near-inertial waves, and $\omega_{1}=1.5 f, \omega_{2}=N$ for super-inertial waves, as discussed above.

The energy exchange between the time-mean and internal wave reservoirs comprises of conversion rates of $\mathrm{KE}, F\left(E_{K}\right)$, and of $\mathrm{APE}, F\left(E_{A}\right)$, defined as

$$
\begin{aligned}
& F\left(E_{K}\right)=-\overline{u_{i}^{\prime} u_{j}^{\prime}} \frac{\partial \bar{u}_{i}}{\partial x_{j}} \\
& =\underbrace{-\left(\overline{u^{\prime} u^{\prime}} \frac{\partial \bar{u}}{\partial x}+\overline{v^{\prime} v^{\prime}} \frac{\partial \bar{v}}{\partial y}+\overline{u^{\prime} v^{\prime}}\left(\frac{\partial \bar{u}}{\partial y}+\frac{\partial \bar{v}}{\partial x}\right)\right)}_{F_{h}\left(E_{K}\right)}-\underbrace{-\left(\overline{u^{\prime} w^{\prime}} \frac{\partial \bar{u}}{\partial z}+\overline{v^{\prime} w^{\prime}} \frac{\partial \bar{v}}{\partial z}\right)}_{F_{v}\left(E_{K}\right)} \\
& -\left(\overline{u^{\prime} w^{\prime}} \frac{\partial \bar{w}}{\partial x}+\overline{v^{\prime} w^{\prime}} \frac{\partial \bar{w}}{\partial y}+\overline{w^{\prime} w^{\prime}} \frac{\partial \bar{w}}{\partial z}\right),
\end{aligned}
$$

and

$$
\begin{aligned}
F\left(E_{A}\right) & =-\left.\overline{u_{i}^{\prime} b^{\prime}} \frac{\partial \bar{b}}{\partial x_{i}} \frac{d z^{*}}{d b}\right|_{b=\bar{b}} \\
& =\underbrace{-\left.\left(\overline{u^{\prime} b^{\prime}} \frac{\partial \bar{b}}{\partial x}+\overline{v^{\prime} b^{\prime}} \frac{\partial \bar{b}}{\partial y}\right) \frac{d z^{*}}{d b}\right|_{b=\bar{b}}}_{F_{h}\left(E_{A}\right)} \underbrace{-\left.\overline{w^{\prime} b^{\prime}} \frac{\partial \bar{b}}{\partial z} \frac{d z^{*}}{d b}\right|_{b=\bar{b}}}_{F_{v}\left(E_{A}\right)} .
\end{aligned}
$$

As convention, $F\left(E_{K}\right), F\left(E_{A}\right)>0(<0)$ indicate energy gain (loss) by the internal wave field. The term $F_{h}\left(E_{K}\right)$ represents $\mathrm{KE}$ conversion rate through horizontal mean shear and strain, whereas 
$F_{v}\left(E_{K}\right)$ represents KE conversion rate through vertical mean shear. The last three terms in Eqn. (15) are presented separately, such that our analysis can be directly compared with previous works (e.g. Cusack et al. 2020) that employ the QG approximation, which assumes that gradients of vertical velocity $w$ are small. The terms $F_{h}\left(E_{A}\right)$ and $F_{v}\left(E_{A}\right)$ represent APE conversion rates through, respectively, horizontal and vertical buoyancy fluxes associated with mean horizontal and vertical buoyancy gradients.

\section{d. Comparison with the $Q G$ simplification}

In this study, we will compute and discuss all of the energy conversion and dissipation rates defined in $\S 3 \mathrm{c}$. However, in this subsection, we will focus specifically on the vertical KE transfer term (i.e., $\left.F_{v}\left(E_{K}\right)\right)$ and horizontal APE transfer term (i.e., $F_{h}\left(E_{A}\right)$ ), which are often combined into the Eliassen-Palm (EP) flux (Eliassen 1960) to highlight the assumptions made by QG theory. The internal wave equation derived by Müller (1976) in the Eulerian framework assumes the time-mean field to be in thermal wind balance, such that the EP flux is defined as

$$
F_{v}\left(E_{K}\right)+F_{h}\left(E_{A}\right)=-\left(\overline{u^{\prime} w^{\prime}}-\frac{f}{N^{2}} \overline{v^{\prime} b^{\prime}}\right) \frac{\partial \bar{u}}{\partial z}-\left(\overline{v^{\prime} w^{\prime}}+\frac{f}{N^{2}} \overline{u^{\prime} b^{\prime}}\right) \frac{\partial \bar{v}}{\partial z},
$$

also assuming per the QG limit that local buoyancy perturbations from the reference density profile are small, i.e., $d z^{*} /\left.d b\right|_{b=\bar{b}} \approx N^{-2}$.

Based on the Wentzel-Kramers-Brillouin-Jeffreys (WKBJ) approximation (Müller 1976) and observations (Ruddick and Joyce 1979), Eqn. (17) can be reduced to an "effective" transfer rate

$$
F_{v}\left(E_{K}\right)+F_{h}\left(E_{A}\right) \approx F_{v}^{e f f}=-\left(1-\frac{f^{2}}{\omega^{2}}\right)\left(\overline{u^{\prime} w^{\prime}} \frac{\partial \bar{u}}{\partial z}+\overline{v^{\prime} w^{\prime}} \frac{\partial \bar{v}}{\partial z}\right) .
$$

This approximation has two important implications: (1) as $\omega \rightarrow f, F_{v}\left(E_{K}\right) \approx-F_{h}\left(E_{A}\right)$, such that there is no energy transfer from vertical shear at the inertial frequency, and (2) at $\omega \gg f, F_{h}\left(E_{A}\right) \approx 0$, such that buoyancy fluxes do not contribute to internal wave energy at higher frequencies. 
However, measurements over Kaena Ridge by Pinkel et al. (2012) have shown contributions from buoyancy fluxes to the total EP flux that are comparable in magnitude to the Reynolds stress terms (i.e., $\overline{u^{\prime} w^{\prime}}, \overline{v^{\prime} w^{\prime}}$ ). More recently, Cusack et al. (2020) found divergence between the effective transfer rate estimate (i.e., Eqn. (18)) and total EP flux (i.e., Eqn. (17)) using mooring data near the bottom in the Southern Ocean.

In the following section, we analyze the spatial patterns of $F_{h}\left(E_{K}\right), F_{v}\left(E_{K}\right)$, and $F_{h}\left(E_{A}\right)$ in our simulations. We assess whether QG theory holds for these energy transfer rates and investigate whether the dynamics of bottom topography-driven flows can explain the above observations. We also consider the role of non-linearity and hydraulic control by comparing across topographic heights.

\section{Conversion between mean and internal wave fields}

\section{a. KE transfer to internal waves}

We first investigate the KE exchange between zero-frequency flow and internal waves, which are separated into the near-inertial and super-inertial frequencies as defined in $\$ 3$. Specifically, we are interested in (1) identifying the regions where the near-inertial waves gain and lose energy, and (2) comparing the near-inertial waves with internal waves at other frequencies. We focus on the near-inertial waves in particular because we previously found in Zemskova and Grisouard (2021) strong non-linear wave-wave interactions between near-inertial waves and lee waves, and that these interactions play an important role in KE dissipation.

We find that the near-inertial waves primarily gain energy through $F_{h}\left(E_{K}\right)$ predominantly via the normal strain term, $-\overline{u^{\prime} u^{\prime}} \partial_{x} \bar{u}$ downstream of the topography and lose energy through the vertical shear term $-\overline{u^{\prime} w^{\prime}} \partial_{z} \bar{u}$, as shown in Figure 5. Because of the nature of one-dimensional topography 
and periodic domain, $\partial_{y}$ gradients for averaged fields are comparatively small. The shear strain term $-\overline{u^{\prime} v^{\prime}} \partial_{x} \bar{v}$ (not shown) has the opposite sign of the normal strain term, but is smaller by approximately a factor of five. The sign of these energy transfers is overall consistent with the observational findings of Cusack et al. (2020). Notably, they recorded short events (order of a few days, similar in duration to $4 t_{I}$ in our study) of vertical energy transfer from internal waves to the time-mean flow (i.e., $F_{v}\left(E_{K}\right)<0$ ) and correspondingly, horizontal energy transfers from the time-mean flow to the internal waves (i.e., $F_{h}\left(E_{K}\right)<0$ ). These patterns can be explained by looking at the CD-filtered velocities, computed using Eqn. (9) at $\omega=0$ (i.e., $\bar{u} / U$ in left panel of Fig. 6) and at $\omega=f$ (i.e., $\tilde{u}_{f} / U$ and $\tilde{w}_{f} / U$ in left and right panels of Fig. 7, respectively). As previously noted in Zemskova and Grisouard (2021), $\bar{u}$ approximately corresponds to lee waves and $\tilde{u}_{f}$ to freely-propagating near-inertial waves, identified from their slopes and wavelengths.

The asymmetry between KE gain by near-inertial waves downstream of topography and their KE loss upstream of topography through horizontal shearing is related to how the flow has to accelerate and slow down as it goes over the bump. While the flow immediately above the topography accelerates as it goes over the obstacle, further above it there is a layer of slowermoving fluid (coined "stagnant" by Winters and Armi (2012)). This layer grows downstream of the topography as the flow dissipates and slows down, such that $\partial_{x} \bar{u}<0$. Because $\overline{u^{\prime} u^{\prime}}$ is positive definite $-\overline{u^{\prime} u^{\prime}} \partial_{x} \bar{u}>0$ and the near-inertial waves are generated because of the horizontal gradient of the zero-frequency flow velocity. Conversely, the near-inertial waves lose KE upstream, where the flow accelerates to go over the obstacle, because $\partial_{x} \bar{u}>0$.

Similar considerations explain how near-inertial waves lose KE to the mean flow slightly aloft of the downstream generation site described above. There, the zero-frequency flow decelerates away from the topography, such that $\partial_{z} \bar{u}<0$ going upward into the stagnant layer from the accelerated near-topography layer. We recall that a freely propagating inertial wave in our mean current $U$ can 
travel along two characteristic slopes $\left(\alpha_{1}, \alpha_{2}\right)$, namely,

$$
\alpha_{1}=0 \quad \text { and } \quad \alpha_{2}=\sqrt{\frac{U k_{2}\left(U k_{2}+2 f\right)}{N^{2}}},
$$

where $\left(k_{1}, k_{2}\right)$ are the two horizontal wavenumbers corresponding to roots of the hydrostatic dispersion relation, and $k_{2}$ is equal to the topographic wavenumber $k$ (see Appendix B in Zemskova and Grisouard (2021)). As such, a freely propagating inertial wave with travelling along the steep characteristic (i.e., $\alpha_{2}$ ) will have a non-zero vertical velocity, unlike an inertial wave travelling along a flat (i.e., $\left.\alpha_{1}\right)$ characteristic. Keeping this fact in mind, as for any internal waves with this orientation, we can expect the real parts of $\tilde{u}_{f}$ and $\tilde{w}_{f}$ for the near-inertial waves propagating on the steep characteristic to be in phase, and the dominant term in vertical KE transfer $\left(F_{v}\left(E_{K}\right)_{f}\right.$, i.e., energy transfer from zero-frequency to near-inertial frequency motions) is $-\overline{u^{\prime} w^{\prime}} \partial_{z} \bar{u}<0$ (not shown), that is, the near-inertial waves lose energy to the zero-frequency flow.

Our results suggest that as non-linearity increases, the hydraulically-controlled leeward side of the topography may host a net transfer of KE from near-inertial waves back to the time-mean flow. Indeed, while both $\left|F_{h}\left(E_{K}\right)_{f}\right|$ and $\left|F_{v}\left(E_{K}\right)_{f}\right|$ increase with increasing $J$, the increase of the vertical transfer term is more substantial. In particular, averaging at a given $H A B$ (cf. right panels of Fig. 5), $\left|F_{h}\left(E_{K}\right)_{f}\right| \gg\left|F_{v}\left(E_{K}\right)_{f}\right|$ for the linear topographic regime simulation $(J=0.6)$. The root cause is that taller topography (and subsequently larger $J$ ) creates greater asymmetry between upstream and downstream flows (cf. Fig. 10 in Zemskova and Grisouard (2021)), increasing $\left|\partial_{x} \bar{u}\right|$. Subsequently, on one hand, at higher $J$, horizontal $\mathrm{KE}$ transfer to near-inertial waves (i.e., $\left.F_{h}\left(E_{K}\right)_{f}\right)$ is not only larger but also takes place closer to the topography downstream, where hydraulic control of the flow becomes more important for greater topographic heights. On the other hand, greater flow acceleration over taller topographies results in a strong increase in the vertical shear, i.e., $\left|\partial_{z} \bar{u}\right|$, downstream close to the topography, which reflects that the difference between the accelerated 
layer and the overlying stagnant layer grows with increasing $J$. In our simulations, the outcome in an overall larger increase for $\left|F_{v}\left(E_{K}\right)_{f}\right|$ compared to that of $\left|F_{h}\left(E_{K}\right)_{f}\right|$. These results suggest that as non-linearity increases, stronger interactions with the zero-frequency flow due to increased hydraulic control near topography may produce stronger near-inertial motions. However, the rate at which near-inertial motions lose energy back to the zero-frequency flow in other places near topography may be even greater. The energy transferred back to the zero-frequency flow can then aid lee wave propagation, akin to the feedback mechanism between lee waves and inertial waves described in Nikurashin and Ferrari (2010a).

KE exchange rates between time-mean flow and super-inertial internal waves are smaller than those between time-mean flow and near-inertial waves (compare Figs. 8 and 5). At $\omega \gg f$, velocity magnitudes and, subsequently, KE spectral density are smaller (cf. Fig. 4). The vertical energy transfer term $F_{v}\left(E_{K}\right)_{S I W}$ is effectively zero. The horizontal energy transfer term, $F_{h}\left(E_{K}\right)_{S I W}$ is also small, consistent with the findings by Cusack et al. (2020) of most of $F_{h}\left(E_{K}\right)$ near bottom at the near-inertial frequencies. For super-inertial motions, the KE transfer is to the internal waves, both from the normal strain and the shear strain terms. The switch in sign in the horizontal shear strain term $\left(-\overline{u^{\prime} v^{\prime}} \partial_{x} \bar{v}\right)$ and the vertical shear term $\left(-\overline{u^{\prime} w^{\prime}} \partial_{z} \bar{u}\right)$ between the near-inertial and superinertial internal waves can be explained by the change from the predominantly counterclockwise rotation at $\omega \approx f$ and clockwise rotation at $\omega>2 f$ (cf. rotary spectra in Fig. 6(b) in Zemskova and Grisouard (2021)). However, overall KE transfers to or from the higher-frequency internal waves are considerably less significant compared with the near-inertial waves.

Interestingly, Cusack et al. (2020) also found vertical KE transfer rates exceeding the horizontal KE transfer rates near the bottom in the Southern Ocean, similar to the results from our simulations with non-linear topographies (i.e., $J \geq 1$ ). It has been previously considered that flows with large $J$ (either because of large $h_{0}$ or small $U$ ) might not play an important role in the internal wave 
energetics due to blocking (e.g., Nikurashin and Ferrari 2010a; Mayer and Fringer 2020), and that such flows would preferentially split to go around a topographic obstacle rather than over it (Nikurashin et al. 2014). However, our energetic analysis for simulations in which the flow is forced to go over tall ridges qualitatively agrees with the near-bottom oceanographic observations. It suggests that there may be situations when the flow is forced to go over a tall topographic obstacle rather than around it, in which case the non-linear dynamics become important and the linear internal wave theory no longer holds. The importance of non-linear dynamics will become even more apparent in the analysis of the buoyancy fluxes in the next subsection.

\section{b. APE transfer to internal waves}

In this section, we investigate whether the linear theory assumptions regarding buoyancy fluxes hold for bottom-driven flows, namely that (1) they balance the vertical shear term at $\omega=f$ and (2) they are small at $\omega \gg f$.

To explain why near-inertial waves can have a direct impact on APE budgets, we recall that freely propagating inertial waves travelling along the steep characteristic (cf. Eqn. (19)) can induce isopycnal displacements in our set-up. The characteristics of the real part of $\tilde{b}_{f}$ align with $\alpha_{2}$ (cf. dashed lines in the middle column of Fig. 7), which is not purely horizontal, whereas the characteristics of the real part of $\tilde{u}_{f}$ are essential horizontal. It suggests that while KE may be preferentially transferred to near-inertial waves with flat characteristics (i.e., $\alpha=0$ ), APE is preferentially transferred to the ones propagating along slanted characteristics (i.e., $\alpha \neq 0$ ) and near-inertial waves may indeed have a footprint on the APE budget.

We find that the QG approximation for the EP flux does not hold, especially for our simulations with taller topography. The left column of Figure 9 shows the APE transfer rates from zerofrequency motions to near-inertial waves due to horizontal buoyancy fluxes (i.e., $\left.F_{h}\left(E_{A}\right)_{f}\right)$. The 
middle column shows $F_{v}\left(E_{K}\right)_{f}+F_{h}\left(E_{A}\right)_{f}$, which according to the expression for the "effective" energy transfer rate shown in Eqn. (18), should be zero. However, we find that $F_{v}\left(E_{K}\right)_{f}$ and $F_{h}\left(E_{A}\right)_{f}$ generally do not cancel each other out. This is true even when we integrate the energy transfer rates along $H A B$ planes (cf. right panels of Fig. 9).

In particular, APE is transferred to the near-inertial waves through buoyancy fluxes both upstream and downstream of the region where the near-inertial waves lose KE through vertical shear. Downstream of the topographic obstacle, $\partial_{x} \bar{b}<0$ (right panels in Fig. 6) as denser water is entrained from below, especially in the larger $J$-regime, in which vigorous overturning occurs (cf. Ri in Fig. 10 of Zemskova and Grisouard (2021)). As shown in Figure 7, in this region, the real parts of $\tilde{b}_{f}$ and $\tilde{w}_{f}$ are out of phase, such that $\overline{w^{\prime} b^{\prime}}<0$, which is consistent with the conversion of KE to APE in the lee of a topographic obstacle previously shown in simulations by Jagannathan et al. (2020). As a result, APE transfers to the near-inertial waves as a result of the horizontal buoyancy fluxes are positive downstream of the topography, i.e., $-\overline{u^{\prime} b^{\prime}} \partial_{x} \bar{b}>0$. Conversely, upstream, $\partial_{x} \bar{b}>0$ and $\tilde{b}_{f}>0$, and the near-inertial waves also gain APE in this region.

Similar to Cusack et al. (2020), we find a mismatch between the "effective" energy transfer rate $F_{v}^{e f f}$ (Eqn. (18)) and the total $F_{v}\left(E_{K}\right)+F_{h}\left(E_{A}\right)$, approximated as the EP flux defined in Eqn. (17). Notably, APE transfer into the near-inertial wave field occurs further above the bottom than KE transfers (cf. right panels of Fig. 9 and 5). Consistent with the near-bottom observations by Cusack et al. (2020), we also find transfers to the internal waves at low frequencies $(\omega \approx f)$ as $F_{h}\left(E_{A}\right)_{f}>0$ even though $F_{v}\left(E_{K}\right)_{f}<0$. These findings further highlight the importance of buoyancy fluxes and non-linear dynamics and shed light on the limitations of the QG approximation.

At super-inertial frequencies $(1.5 f \leq \omega \leq N)$, APE transfer due to buoyancy fluxes is indeed smaller than the KE transfer due to vertical shear for small topographies (cf. Fig. 10(a-d)). However, in the more non-linear regime (i.e., $J=2)$ in particular, contributions from $F_{h}\left(E_{A}\right)$ and $F_{v}\left(E_{K}\right)$ are 
approximately equal, and the APE transfer augments the vertical KE transfer from internal waves to the zero-frequency flow. In particular, up to $H A B=0.2$, the approximation of the EP flux by $F_{v}^{\text {eff }}$ is no longer valid, highlighting the importance of non-linear interactions near the topography, as discussed in Cusack et al. (2020). Our results from simulations with non-linear topographies $(J>1)$ are consistent with their observations, in particularly negative $F_{h}\left(E_{A}\right)+F_{v}\left(E_{K}\right)$ at super-inertial frequencies in contrast with positive transfer rates at near-inertial frequencies.

In this section, we described a qualitative agreement between our non-linear simulations and the near-bottom ocean observations with respect to the KE and APE transfer rates. In the next section, we will investigate the effect of such non-linear dynamics on KE and APE dissipation rates, and subsequently, mixing efficiency.

\section{Mixing efficiency}

Average mixing efficiency over a given volume $V$ is defined as the ratio of irreversible mixing to the total energy sink (Peltier and Caulfield 2003), namely,

$$
\eta_{V}=\frac{\int_{V} D\left(E_{A}\right) d V}{\int_{V} D\left(E_{A}\right) d V+\int_{V} D\left(E_{K}\right) d V} .
$$

Specifically, in order to investigate the role of topography-driven dynamics, we compute $\eta_{V}$ over four volumes bounded in terms of heights above the bottom: $H A B<0.1, H A B \in[0.1,0.2)$, $H A B \in[0.2,0.3)$, and $H A B \in[0.3,0.4)$. We can also define mixing efficiency locally to analyze its spatial distribution, such that:

$$
\eta=\frac{D\left(E_{A}\right)}{D\left(E_{A}\right)+D\left(E_{K}\right)} .
$$

It is important to note that $\frac{1}{V} \int_{V} \eta d V \neq \eta_{V}$. In broad strokes, the distribution of mixing efficiency reflects the competition between KE and APE dissipation rates, which we describe in detail below. 
For all simulations, enhanced KE dissipation is sustained by energy exchanges with near-inertial motions that result from resonant wave-wave interactions as discussed in Zemskova and Grisouard (2021). In Figure 2, we qualitatively show that $D\left(E_{K}\right)$ remains large wherever the non-linear forcing $\left|\Lambda_{f}\right|$ is also arbitrarily not small. For all simulations, $\Lambda_{f}>0$ near the topography where the near-inertial motions are generated, and those regions are also characterized by elevated $D\left(E_{K}\right)$. Looking further away from the topography, for $J=1$, for example, there is another peak with $\Lambda_{f}>0$ around $H A B=0.2$, and high $D\left(E_{K}\right)$ rates are also sustained up to this height. However, for $H A B>0.2, \Lambda_{f} \rightarrow 0$ and $D\left(E_{K}\right)$ is also small. Similarly, for $J=0.6, D\left(E_{K}\right)$ is small above $H A B \approx 0.12$, where $\left|\Lambda_{f}\right| \approx 0$. In contrast, for $J=2$, both $D\left(E_{K}\right)$ and $\left|\Lambda_{f}\right|$ remain large far above the topography. While we are unable to determine the precise cutoff value for $\left|\Lambda_{f}\right|$, our results suggest that the vertical extent of a region where KE dissipation rates are elevated is correlated with the region where non-linear wave-wave interactions with near-inertial motions are significant. The distribution of mixing efficiency $\eta_{V}$ above the bottom in Figure 11(a) shows distinct patterns for small, linear topographies $(J=0.6)$, for which mixing efficiency increases with $H A B$, and tall, more non-linear topographies $(J=2)$, for which mixing efficiency decreases with $H A B$. Local mixing efficiency $\eta$ distributions in Figure 12(a,c) show a predominantly lee wave-like spatial pattern for small topography $(J=0.6)$, as expected from linear theory, whereas wave-like structures are less apparent and a signal of hydraulics downstream of the topography is prominent in the more non-linear $J=2$ case. These patterns reflect the effect of topographic height on the competition between the KE and APE dissipation rates. For small topographies, the local Richardson number remains above zero, there are no overturns near the topography (cf. Fig. 10(d) in Zemskova and Grisouard (2021)), and mixing rate is low (Fig. 12(a)). However, resonant non-linear wave-wave interactions likely enhance kinetic energy dissipation as shown in Figure 2(a) and volume-averaged mixing efficiency $\eta_{V}$ is small near the topography $(H A B<0.2)$. It reflects that the region of 
strong $D\left(E_{K}\right)$ is confined to $H A B \approx 0.15$ (cf. Figs. 2(a) and 11(b)) limited by the vertical extent of significant non-linear wave-wave interactions. On the other hand, buoyancy gradients are not eroded by overturns, so mixing rate (i.e., $D\left(E_{A}\right)$ in Fig. 11(b)) starts small near the topography but decreases less sharply with $H A B$ than $D\left(E_{K}\right)$ and, as a result, $\eta_{V}$ increases with $H A B$.

In contrast, the region close to topography for tall topographic regimes (e.g., $J=2)$ is characterized by a high degree of instability and overturning, in part due to loss of balance and hydraulic control (Zemskova and Grisouard 2021). Strong overturns can be characterized by large negative $\overline{w^{\prime} b^{\prime}}$ term, which signifies internal wave energy transfer from KE to APE, as denser waters are brought upwards and lighter waters are brought downwards. Near the bottom $(H A B<0.2$, Fig. 11(c)), this term is negative for all simulations, and increases in magnitude with $J$. While $D\left(E_{K}\right)$ is also high in this region, mixing rate is comparatively more pronounced, owing to large buoyancy gradients. These elevated mixing rates are evident in both the volume-averaged $\eta_{V}$ for $H A B \in[0.1,0.2)$ (cf. Fig. 11(a)), and local $\eta$, in particular downstream of topography where hydraulics are important (cf. Fig. 12(c)). ${ }^{1}$ Over this vertical extent, internal waves continue to gain APE from the KE reservoir in simulation with $J=2$, whereas the magnitude of $\overline{w^{\prime} b^{\prime}}$ drops close to zero for simulations with smaller topographies (Fig. 11(c)). The relative importance of the exchange between internal wave KE and APE also appears in the KE and APE spectra in Fig. 4: KE decreases and APE increases with $H A B$ for $J=2$ (and to lesser extent $J=0.1$ ), whereas for $J=0.6$, changes in KE and APE spectra with $H A B$ are less prominent.

It is noteworthy that super-inertial internal waves are responsible for a large portion of APE dissipation, especially compared with the near-inertial waves (cf. Fig. 13(a-b)). We can estimate

\footnotetext{
${ }^{1}$ It is important to note that mixing rates and mixing efficiency directly above the topography are very small for this simulation with $J=2$. It happens both because of topographic blocking of the flow and because we do not restore the stratification in these simulations, such that there is a bottom layer with reduced buoyancy frequency, similar to other previous studies of flows over periodic hills (e.g. Klymak 2018; Mayer and Fringer 2020).
} 
the rate of APE transfer from near-inertial to super-inertial internal waves as

$$
F\left(E_{A}^{f}, E_{A}^{S I W}\right)=-\left.\left(\overline{u^{\prime} b^{\prime}} \frac{\partial \tilde{b}_{f}}{\partial x}+\overline{w^{\prime} b^{\prime}} \frac{\partial \tilde{b}_{f}}{\partial z}\right) \frac{d z^{*}}{d b}\right|_{b=\tilde{b}_{f}},
$$

which is analogous to the APE transfer term from zero-frequency flow to internal waves in Eqn. (16), ignoring $\partial_{y}$ term, which is comparatively small on average because of the domain periodicity and 1D nature of the topography. For $J=2$, in particular, this term is large and positive over $H A B \in[0.1,0.3)$ (cf. Fig. 11(d)), such that super-inertial internal waves gain APE. This result points to the importance of a forward energy cascade to sustain high irreversible mixing rates and subsequently high mixing efficiency in this region.

However, strong mixing events near the topography erode both horizontal and vertical buoyancy gradients. For instance, the wave-like patterns of both $\bar{b}$ and $\tilde{b}_{f}$ appear broken up at $J=2$ (cf. (Fig. 6(f) and Fig. 7(h)) compared with the more coherent structures in the simulations with smaller topographies (cf. Fig. 6(b,d) and Fig. 7(b,e)). As such, for $J=2$, mixing rates, which are functions of buoyancy gradients, sharply decrease with height above $H A B=0.2$ by two orders of magnitude (Fig. 11(b)). Yet, higher kinetic energy dissipation rates are sustained away from the topography (Fig. 2(c)) owing to the near-inertial motions that result from resonant wave-wave interactions. As a result, mixing efficiency decreases away from the bottom in the simulations with non-linear topography $(J=2)$.

Mixing efficiency for the transitional topographic regime $J=1$ at intermediate depths $H A B \in$ $[0.1,0.3)$ unsurprisingly lies in between the small and large topographic regimes (cf. Fig. 11(a)). However, $\eta_{V}$ is higher both near the topography $(H A B<0.1)$ and remarkably far away from the topography $(H A B \in[0.3,0.4))$ for $J=1$ compared with $J=0.6$ and $J=2$ simulations. In the $J=1$ simulation near the topography, there is some overturning and turbulent mixing, and energy transfer from KE to APE for the internal waves (Fig. 11(c)). However, because overturning is 
not as vigorous as in the higher topography simulation $(J=2)$, buoyancy gradients are not eroded as much, and there is APE transfer to super-inertial internal waves both from zero-frequency motions (Fig. 10(c)) and from near-inertial motions (Fig. 11(d)). As such, close to topography, irreversible mixing rates for the $J=1$ and $J=2$ simulations are similar (Fig. 13(a-b)), whereas KE dissipation rates for $J=1$ simulation are $1-2$ orders of magnitude smaller than the $J=2$ simulation (Fig. 13(c-d)).

In our simulations, we observe that $\mathrm{KE}$ is dissipated more by the zero-frequency flow (e.g., lee waves) than internal waves (Fig. 13(c)), whereas APE dissipation rates from the zero-frequency flow and internal waves is approximately equal, especially for $J=1,2$ (Fig. 13(a)). KE dissipation rates are driven by the non-linear wave-wave interactions, primarily between the near-inertial waves and zero-frequency flow, which are small above $H A B=0.2$ for the $J=1$ simulation (Fig. 2(b)). However, APE dissipation rates, in particular of super-inertial internal waves, remain high even at $H A B>0.3$ (Fig. 13(b)), as buoyancy gradients and buoyancy fluxes remain large enough that APE is transferred to higher frequencies (Fig. 11(d)). Because of such balance (low KE dissipation and high APE dissipation rates), $\eta_{V}$ over $H A B \in[0.3,0.4)$ is larger in the simulation with $J=1$ compared with other simulations. Interestingly, for $J=0.6$ and $J=1$, the region of increased mixing efficiency $\eta_{V}$ lies above the region of increased energy transfer to super-inertial internal waves (cf. Fig. 11(a,d)). It is possible that because of larger group velocities at higher frequencies (all else being equal, per the internal wave dispersion relation), super-inertial internal waves can propagate vertically faster and displace isopycnals farther away from their generation site. This or some other mechanism for non-local transport and dissipation of APE could be further explored in a follow-up study.

Our results highlight that although both KE and APE dissipation rates increase with topographic height, their spatial distributions are significantly different, such that the effects of topography on 
mixing efficiency are non-trivial. In previous sections, we showed that energetics of the flow over more non-linear, tall topographies (i.e., $J=2$ ) could explain the energy transfer rates observed near the bottom in the Southern Ocean by Cusack et al. (2020). As such, the differences in patterns of mixing efficiency between non-linear and linear cases may be important for future studies of ocean mixing.

\section{Discussion and summary}

In this study, we examine the effects of topographic height on the energetics of stratified flow over lee wave radiative topography through idealized numerical simulations. Specifically, we focus on 1) the energy transfer between the non-geostrophic component of the time-mean flow and internal waves with frequencies between $\sim f$ and $N$, and 2) KE and APE dissipation rates. We find a number of differences between a linear regime (short topography) and a more non-linear regime (tall topography), and, interestingly, a qualitative agreement between the tall topography $(J=2)$ simulation and observational measurements by Cusack et al. (2020) in the Southern Ocean. Our findings suggest that non-linear dynamics may play an important role near the ocean bottom, especially over tall, lee-wave radiative topography.

\section{a. Energy transfer rates}

For our simulation with non-linear taller topography $(J=2)$, we find the following main similarities with the observed near-bottom energy transfer rates reported in Cusack et al. (2020):

1. net KE transfer from the internal waves to the time-mean flow due to the vertical shear is in magnitude approximately equal to or exceeding, but has opposite sign to the horizontal KE transfer, which facilitates energy transfer to the internal waves through horizontal shear and strain; 
2. near-inertial waves are responsible for most of the horizontal KE transfer rates with minimal contributions from super-inertial internal waves;

3. buoyancy fluxes of super-inertial $(\omega>1.5 f)$ internal waves play an important role in energy transfers from the internal waves.

In contrast, the simulation with shorter linear topography $(J=0.6)$ has vertical $\mathrm{KE}$ transfer rates that are negligible compared with the horizontal $\mathrm{KE}$ transfer rates. It also has negligible contribution to the energy transfer rates from the buoyancy flux terms. Vertical KE transfer rates, in particular, increase with topographic height due to larger vertical shear of the time-mean flow (e.g., $\partial_{z} \bar{u}$ ) near the topography because the flow has to accelerate and decelerate more while going over the obstacle.

The derivation of EP flux (Eliassen 1960), which combines KE transfer due to vertical shears and APE transfer from horizontal buoyancy fluxes (cf. Eq. (17)), and the further "effective" transfer rate approximation by Ruddick and Joyce (1979) (cf. Eq. (18)) both rely on the geostrophic balance of the mean flow and linear internal wave dynamics. Cusack et al. (2020) attributed discrepancy between the observations and such approximations to the non-linear dynamics, namely large $J$ in the study area. Thus, it is perhaps not surprising that we find an agreement between our simulation with tall non-linear topography and their observations. However, notably, in the Eulerian framework, near-bottom time-mean flow can also include non-geostrophic components, for instance, steady lee waves. Previous studies (e.g., Nikurashin and Ferrari 2010a; Zemskova and Grisouard 2021) found the interaction between lee waves and near-inertial waves to be important for bottom-driven flows. In the energetic analysis of our simulations, we indeed find that the wave-wave interactions between the non-geostrophic time-mean flow (primarily lee waves) and internal waves (with frequencies between $f$ and $N$ ) produce energy transfer rates that qualitatively agree with the observations. 
Our findings have two main implications. First, the "effective" transfer rate approximation has been often applied to parameterize the effective vertical viscosity by combining the contribution of vertical stresses and buoyancy fluxes, and then used to estimate dissipation rates (e.g., Ferrari and Wunsch 2009; Polzin 2010). This approximation implies that near-inertial motions do not contribute to the vertical energy transfer, and that KE exchange plays a more important role than APE exchange. Applying this approximation to observational measurements, vertical viscosity have been computed to be at least 3 orders of magnitude smaller than horizontal viscosity (e.g., Watson 1985; Polzin 2010). As such, vertical KE transfer has previously been considered negligible and excluded from ocean energy budget analyses (e.g., Storch et al. 2012; Jing et al. 2018). Our analysis suggests that in regions of non-linear and non-geostrophic flows, for instance near tall bottom topography where strong near-inertial motions are generated, such approximation may no longer hold. In these regions, contribution from near-inertial motions and buoyancy fluxes to vertical viscosity parameterizations may need to be included, especially for observational studies conducted in the Eulerian framework, where stationary lee waves are part of the zero-frequency (time-mean) flow.

Second, it has been previously suggested that flow over two-dimensional topography may preferentially go around a tall topographic obstacle rather than over it, such that the energy flux into the internal waves is reduced for sufficiently non-linear topographies (e.g., $J>0.7$ in Nikurashin et al. (2014)). However, in this study, we find a qualitative agreement in energy transfer rates between the ocean observations and our simulation with non-linear topography $(J=2)$, but not our simulation with linear topography $(J=0.6)$. Because of the anisotropic nature of the abyssal hills, it may be the case that the flow is forced to go over some topographic obstacles with $J>1$. As pointed out by Mayer and Fringer (2020), due to this anisotropic nature, in some regions the topography may be better approximated as a series of ridges rather than seamounts. Notably, strong contributions 
to energy transfer rates from horizontal buoyancy fluxes have been previously reported in flows over ridges (e.g., Pinkel et al. 2012). As such, it is possible that simplification to one-dimensional topography is valid for idealized process studies of flow over bottom topography, and that hydraulic control and other non-linear processes that become important for taller topographies indeed contribute to ocean energetics.

Because our background geostrophic flow is uniform, our analysis differs from previous studies (Shakespeare and McC. Hogg 2018; Kunze and Lien 2019) that considered energy loss or gain of the internal waves to the mean geostrophic shear. The presence of such shear would further complicate the energy exchanges between various energy reservoirs, and deserves a separate study (Cynthia $\mathrm{Wu}$, personal communication). Nonetheless, the results from this study can provide an insight into the energy transfers between lee waves and other internal waves, in particular near-inertial waves, which are all generated as a result of flow-topography interactions.

\section{b. KE and APE dissipation}

In our simulations, the rates of both KE dissipation and APE dissipation (i.e., irreversible mixing) increase with height of the topographic obstacle, but their spatial distributions differ. As the topographic height increases, mixing is enhanced locally, close to the topography, especially in the region of wave breaking and convective overturns. This is consistent with the assumption of locally generated mixing used to estimate global mixing efficiency and overturning rates in Mashayek et al. (2017b). KE dissipation, on the other hand, can be sustained further above the topography as $J$ increases through increased non-linear wave-wave interactions. The vertical extent of these non-linear resonant interactions, especially those involving steady lee waves and upward propagating near-inertial waves, increases with topographic height, as instabilities arising in the hydraulically controlled region near topography help generate stronger near-inertial waves. As a 
result, mixing efficiency, which reflects the competing effects of KE and APE dissipation rates, increases away from bottom for flows over shorter topographies, but it is maximized close to the bottom for flows over taller topographies.

Our results add new evidence to the volume of previous literature showing the variability of mixing efficiency in different environments (e.g., Moum 1996; Scotti and White 2011; Salehipour and Peltier 2015; Chalamalla and Sarkar 2015; Mashayek et al. 2017a; Sohail et al. 2019). Interestingly, sufficiently away from the shorter topography $(J=0.6 ; H A B>0.2$, which is approximately $800 \mathrm{~m}$ ), volume-averaged mixing efficiency $\eta_{V}$ is around $1 / 6$, which corresponds to the canonical value for the flux coefficient $\Gamma=0.2$ (cf. Eqn. (2)). As such, for flows over linear topography, away from the bottom-driven local effects, previous estimates of turbulent diffusion coefficient $\kappa_{\text {tur }}$ may be valid. However, for taller topographies, the flux coefficient may be much smaller (e.g., at $H A B=0.3$, for $J=2, \eta_{V} \sim 0.05$ and $\Gamma=0.053$ ) or larger (e.g., at $H A B=0.3$, for $J=1, \eta_{V} \sim 0.3$ and $\Gamma=0.43$ ). If flows over non-linear topographies indeed have important contributions to ocean energetics, as suggested in our study, $\Gamma=0.2$ may underestimate or overestimate $\kappa_{t u r b}$ depending on the height of a topographic obstacle and, subsequently, miscalculate the rate of abyssal overturning in global models.

Because of the highly idealized set-up of this process study, we are unable to account for all processes that undoubtedly affect dissipation and mixing rates close to and away from bottom topography in the ocean. For instance, the set-up does not allow for the flow to go around the topography (Nikurashin et al. 2014) or include the effects of far-field propagation and remote dissipation of bottom-radiated waves (Zheng and Nikurashin 2019). Because the topography in our simulations has a fixed width, we also have not captured variability over the full $J-\chi$ spectrum, which was shown to be important by Mayer and Fringer (2020). Furthermore, the rate of turbulent mixing may be underestimated, especially in the more non-linear topographic regimes, 
due to the loss of stratification near topography. In regions of the ocean where stratified fluid is continuously supplied, mixing efficiency may be indeed larger. As such, the specific values of $\eta_{V}$ (and subsequently, $\Gamma$ ) computed in this study may not be directly applicable to the ocean without further numerical studies accounting for some, if not all, of these processes. Nonetheless, our results can provide insights into the differences between the energetics of bottom-driven flows over short and tall abyssal hills that may need to be considered for parameterizations of turbulent viscosity and diffusivity coefficients.

Acknowledgments. We acknowledge the financial support of the Natural Sciences and Engineering Research Council of Canada (NSERC) [RGPIN-2015-03684]. Computations were performed on the Niagara supercomputer at the SciNet HPC Consortium. SciNet is funded by: the Canada Foundation for Innovation; the Government of Ontario; Ontario Research Fund - Research Excellence; and the University of Toronto (Loken et al. 2010; Ponce et al. 2019).

Data availability statement. The data generated for these simulations exceeds 5TB and cannot be easily distributed. However, we provided all Nek5000 codes necessary to run the simulations presented in this paper and all Python codes used for post-processing at https://github.com/ bzemskova/bottom_topography_flow.

\section{References}

Alford, M. H., and Coauthors, 2015: The formation and fate of internal waves in the south china sea. Nature, 521 (7550), 65-69.

Bell, T., 1975: Lee waves in stratified flows with simple harmonic time dependence. Journal of Fluid Mechanics, 67 (4), 705-722. 
Brearley, J. A., K. L. Sheen, A. C. Naveira Garabato, D. A. Smeed, and S. Waterman, 2013: Eddy-induced modulation of turbulent dissipation over rough topography in the southern ocean. Journal of physical oceanography, 43 (11), 2288-2308.

Brown, E. D., and W. B. Owens, 1981: Observations of the horizontal interactions between the internal wave field and the mesoscale flow. Journal of Physical Oceanography, 11 (11), $1474-1480$.

Caulfield, C., 2021: Layering, instabilities, and mixing in turbulent stratified flows. Annual Review of Fluid Mechanics, 53.

Chalamalla, V. K., and S. Sarkar, 2015: Mixing, dissipation rate, and their overturn-based estimates in a near-bottom turbulent flow driven by internal tides. Journal of Physical Oceanography, 45 (8), 1969-1987.

Cusack, J. M., J. A. Brearley, A. C. Naveira Garabato, D. A. Smeed, K. L. Polzin, N. Velzeboer, and C. J. Shakespeare, 2020: Observed eddy-internal wave interactions in the southern ocean. Journal of Physical Oceanography, 50 (10), 3043-3062.

De Lavergne, C., G. Madec, J. Le Sommer, A. G. Nurser, and A. C. Naveira Garabato, 2016: The impact of a variable mixing efficiency on the abyssal overturning. Journal of Physical Oceanography, 46 (2), 663-681.

Eliassen, A., 1960: On the transfer of energy in stationary mountain waves. Geophy. Publ., 22, $1-23$

Ferrari, R., and C. Wunsch, 2009: Ocean circulation kinetic energy: Reservoirs, sources, and sinks. Annual Review of Fluid Mechanics, 41. 
Fischer, P. F., J. W. Lottes, and S. G. Kerkemeier, 2008: Nek5000 web page. URL http://nek5000. mcs.anl.gov.

Grisouard, N., and L. N. Thomas, 2015: Critical and near-critical reflections of near-inertial waves off the sea surface at ocean fronts. Journal of Fluid Mechanics, 765, 273.

Hu, Q., and Coauthors, 2020: Cascade of internal wave energy catalyzed by eddy-topography interactions in the deep south china sea. Geophysical Research Letters.

Hughes, G. O., A. M. C. Hogg, and R. W. Griffiths, 2009: Available potential energy and irreversible mixing in the meridional overturning circulation. Journal of Physical Oceanography, 39 (12), $3130-3146$.

Jagannathan, A., K. B. Winters, and L. Armi, 2020: The effect of a strong density step on blocked stratified flow over topography. Journal of Fluid Mechanics, $\mathbf{8 8 9}$.

Jing, Z., P. Chang, S. DiMarco, and L. Wu, 2018: Observed energy exchange between lowfrequency flows and internal waves in the gulf of mexico. Journal of Physical Oceanography, 48 (4), 995-1008.

Klymak, J. M., 2018: Nonpropagating form drag and turbulence due to stratified flow over largescale abyssal hill topography. Journal of Physical Oceanography, 48 (10), 2383-2395.

Klymak, J. M., S. M. Legg, and R. Pinkel, 2010: High-mode stationary waves in stratified flow over large obstacles. Journal of Fluid Mechanics, 644, 321-336.

Klymak, J. M., R. Pinkel, and L. Rainville, 2008: Direct breaking of the internal tide near topography: Kaena ridge, hawaii. Journal of Physical Oceanography, 38 (2), 380-399.

Kunze, E., and R.-C. Lien, 2019: Energy sinks for lee waves in shear flow. Journal of Physical Oceanography, 49 (11), 2851-2865. 
Legg, S., 2021: Mixing by oceanic lee waves. Annual Review of Fluid Mechanics, 53.

Legg, S., and J. Klymak, 2008: Internal hydraulic jumps and overturning generated by tidal flow over a tall steep ridge. Journal of Physical Oceanography, 38 (9), 1949-1964.

Liang, X., and A. M. Thurnherr, 2012: Eddy-modulated internal waves and mixing on a midocean ridge. Journal of physical oceanography, 42 (7), 1242-1248.

Loken, C., and Coauthors, 2010: SciNet: Lessons Learned from Building a Powerefficient Top-20 System and Data Centre. J. Phys. Conf. Ser., 256, 012026, doi:10. 1088/1742-6596/256/1/012026, URL http://stacks.iop.org/1742-6596/256/i=1/a=012026?key= crossref.460dd0a7bf20e10e76a0c5799b82a3d5.

Mashayek, A., R. Ferrari, S. Merrifield, J. Ledwell, L. St Laurent, and A. N. Garabato, 2017a: Topographic enhancement of vertical turbulent mixing in the southern ocean. Nature communications, $8(\mathbf{1}), 1-12$.

Mashayek, A., H. Salehipour, D. Bouffard, C. Caulfield, R. Ferrari, M. Nikurashin, W. Peltier, and W. Smyth, 2017b: Efficiency of turbulent mixing in the abyssal ocean circulation. Geophysical Research Letters, 44 (12), 6296-6306.

Mayer, F. T., and O. B. Fringer, 2020: Improving nonlinear and nonhydrostatic ocean lee wave drag parameterizations. Journal of Physical Oceanography, 50 (9), 2417-2435.

Mercier, M. J., N. B. Garnier, and T. Dauxois, 2008: Reflection and diffraction of internal waves analyzed with the hilbert transform. Physics of Fluids, 20 (8), 086601.

Moum, J. N., 1996: Efficiency of mixing in the main thermocline. Journal of Geophysical Research: Oceans, 101 (C5), 12 057-12069. 
Müller, P., 1976: On the diffusion of momentum and mass by internal gravity waves. Journal of Fluid Mechanics, 77 (4), 789-823.

Munk, W., and C. Wunsch, 1998: Abyssal recipes ii: Energetics of tidal and wind mixing. Deep-sea research. Part I, Oceanographic research papers, 45 (12), 1977-2010.

Musgrave, R., J. MacKinnon, R. Pinkel, A. Waterhouse, J. Nash, and S. Kelly, 2017: The influence of subinertial internal tides on near-topographic turbulence at the mendocino ridge: Observations and modeling. Journal of Physical Oceanography, 47 (8), 2139-2154.

Nikurashin, M., and R. Ferrari, 2010a: Radiation and dissipation of internal waves generated by geostrophic motions impinging on small-scale topography: Application to the southern ocean. Journal of Physical Oceanography, 40 (9), 2025-2042.

Nikurashin, M., and R. Ferrari, 2010b: Radiation and dissipation of internal waves generated by geostrophic motions impinging on small-scale topography: Theory. Journal of Physical Oceanography, 40 (5), 1055-1074.

Nikurashin, M., and R. Ferrari, 2013: Overturning circulation driven by breaking internal waves in the deep ocean. Geophysical Research Letters, 40 (12), 3133-3137.

Nikurashin, M., R. Ferrari, N. Grisouard, and K. Polzin, 2014: The impact of finite-amplitude bottom topography on internal wave generation in the southern ocean. Journal of Physical Oceanography, 44 (11), 2938-2950.

Nikurashin, M., and G. Vallis, 2011: A theory of deep stratification and overturning circulation in the ocean. Journal of Physical Oceanography, 41 (3), 485-502.

Peltier, W., and C. Caulfield, 2003: Mixing efficiency in stratified shear flows. Annual review of fluid mechanics, 35 (1), 135-167. 
Pinkel, R., L. Rainville, and J. Klymak, 2012: Semidiurnal baroclinic wave momentum fluxes at kaena ridge, hawaii. Journal of physical oceanography, 42 (8), 1249-1269.

Polzin, K. L., 2009: An abyssal recipe. Ocean Modelling, 30 (4), 298-309.

Polzin, K. L., 2010: Mesoscale eddy-internal wave coupling. part ii: Energetics and results from polymode. Journal of physical oceanography, 40 (4), 789-801.

Ponce, M., and Coauthors, 2019: Deploying a Top-100 Supercomputer for Large Parallel Workloads. Proc. Pract. Exp. Adv. Res. Comput. Rise Mach., ACM, New York, NY, USA, 1-8, doi:10.1145/3332186.3332195, URL https://dl.acm.org/doi/10.1145/3332186.3332195.

Ruddick, B. R., and T. M. Joyce, 1979: Observations of interaction between the internal wavefield and low-frequency flows in the north atlantic. Journal of Physical Oceanography, 9 (3), 498-517.

Salehipour, H., and W. Peltier, 2015: Diapycnal diffusivity, turbulent prandtl number and mixing efficiency in boussinesq stratified turbulence. Journal of Fluid Mechanics, 775, 464.

Scott, R., J. Goff, A. Naveira Garabato, and A. Nurser, 2011: Global rate and spectral characteristics of internal gravity wave generation by geostrophic flow over topography. Journal of Geophysical Research: Oceans, 116 (C9).

Scotti, A., and B. White, 2011: Is horizontal convection really "non-turbulent?". Geophysical Research Letters, 38 (21).

Scotti, A., and B. White, 2014: Diagnosing mixing in stratified turbulent flows with a locally defined available potential energy. Journal of fluid mechanics, 740, 114.

Shakespeare, C. J., and A. M. Hogg, 2017: Spontaneous surface generation and interior amplification of internal waves in a regional-scale ocean model. Journal of Physical Oceanography, 47 (4), 811-826. 
Shakespeare, C. J., and A. McC. Hogg, 2018: The life cycle of spontaneously generated internal waves. Journal of Physical Oceanography, 48 (2), 343-359.

Sheen, K., and Coauthors, 2013: Rates and mechanisms of turbulent dissipation and mixing in the southern ocean: Results from the diapycnal and isopycnal mixing experiment in the southern ocean (dimes). Journal of Geophysical Research: Oceans, 118 (6), 2774-2792.

Sohail, T., C. A. Vreugdenhil, B. Gayen, and A. M. Hogg, 2019: The impact of turbulence and convection on transport in the southern ocean. Journal of Geophysical Research: Oceans, $124(6), 4208-4221$.

Storch, J.-S. v., C. Eden, I. Fast, H. Haak, D. Hernández-Deckers, E. Maier-Reimer, J. Marotzke, and D. Stammer, 2012: An estimate of the lorenz energy cycle for the world ocean based on the storm/ncep simulation. Journal of physical oceanography, 42 (12), 2185-2205.

Sun, O. M., and R. Pinkel, 2012: Energy transfer from high-shear, low-frequency internal waves to high-frequency waves near kaena ridge, hawaii. Journal of physical oceanography, 42 (9), $1524-1547$.

Waterhouse, A. F., and Coauthors, 2014: Global patterns of diapycnal mixing from measurements of the turbulent dissipation rate. Journal of Physical Oceanography, 44 (7), 1854-1872.

Waterman, S., A. C. Naveira Garabato, and K. L. Polzin, 2013: Internal waves and turbulence in the antarctic circumpolar current. Journal of Physical Oceanography, 43 (2), 259-282.

Waterman, S., K. L. Polzin, A. C. Naveira Garabato, K. L. Sheen, and A. Forryan, 2014: Suppression of internal wave breaking in the antarctic circumpolar current near topography. Journal of physical oceanography, 44 (5), 1466-1492. 
Watson, K. M., 1985: Interaction between internal waves and mesoscale flow. Journal of physical oceanography, 15 (10), 1296-1311.

Whalen, C. B., J. A. MacKinnon, and L. D. Talley, 2018: Large-scale impacts of the mesoscale environment on mixing from wind-driven internal waves. Nature Geoscience, 11 (11), 842-847.

Winters, K. B., and L. Armi, 2012: Hydraulic control of continuously stratified flow over an obstacle. Journal of fluid mechanics, 700, 502-513.

Winters, K. B., and E. A. D’Asaro, 1996: Diascalar flux and the rate of fluid mixing. Journal of Fluid Mechanics, 317, 179-193.

Winters, K. B., P. N. Lombard, J. J. Riley, and E. A. D’Asaro, 1995: Available potential energy and mixing in density-stratified fluids. Journal of Fluid Mechanics, 289, 115-128.

Wunsch, C., and R. Ferrari, 2004: Vertical mixing, energy, and the general circulation of the oceans. Annu. Rev. Fluid Mech., 36, 281-314.

Yang, L., M. Nikurashin, A. M. Hogg, and B. M. Sloyan, 2018: Energy loss from transient eddies due to lee wave generation in the southern ocean. Journal of Physical Oceanography, 48 (12), $2867-2885$.

Zemskova, V. E., and N. Grisouard, 2021: Near-inertial dissipation due to stratified flow over abyssal topography.

Zemskova, V. E., B. L. White, and A. Scotti, 2015: Available potential energy and the general circulation: Partitioning wind, buoyancy forcing, and diapycnal mixing. Journal of Physical Oceanography, 45 (6), 1510-1531.

Zemskova, V. E., B. L. White, and A. Scotti, 2020: Energetics of a rotating wind-forced horizontal convection model of a reentrant channel. Journal of Physical Oceanography. 
Zheng, K., and M. Nikurashin, 2019: Downstream propagation and remote dissipation of internal waves in the southern ocean. Journal of Physical Oceanography, 49 (7), 1873-1887. 


\section{LIST OF FIGURES}

Fig. 1. Domain set-up for all simulations with sinusoidal topographic bump (upper part of the domain cropped). Overlayed is a snapshot of the flow for experiment $(\chi, J)=(0.16,2)$ at $t=6.75 t_{I}$ to highlight a breaking event downstream of the topography.. Color: normalized perturbation velocity $u / U$; black contours: isopycnals. Topography is homogeneous in $y$ and the domain is periodic in $x$ and $y$.

Fig. 2. (Left) Hovmöller diagram of the normalized total kinetic energy dissipation $\left(\log _{10}\left(E_{K} / U^{2}\right)\right)$ horizontally averaged and plotted in terms of height above the bottom (HAB) and (right) horizontally-averaged non-linear forcing for the near-inertial frequency motions, $\Lambda_{f}$ defined in Eqn. (10) computed over the last $4 t_{I}$. (a) $J=0.6$, (b) $J=1$, and (c) $J=2$.

Fig. 3. Energy budget diagram illustrating KE and APE exchanges between the time-mean flow and internal waves, and dissipation rates of the time mean flow and internal waves. KE and APE are defined in Eqns. (7) and (8), respectively. KE and APE exchange rates are defined in Eqns. (15) and (16), respectively. The dissipation rates of KE and APE are defined in Eqns. (13) and (14), respectively. When a term on an arrow is positive (negative), energy goes along (opposite to) the direction of said arrow.

Fig. 4. (a,c) Normalized kinetic energy $\left(E_{K}\right)$ spectra, and (b,d) available potential energy $\left(E_{A}\right)$ spectra, excluding the background geostrophic flow, averaged at $H A B=0.1,0.2,0.3$. (Top) simulation with linear topography $J=0.6$, and (bottom) simulation with non-linear topography $J=2$. Spectra are computed over the last $4 t_{I}$ of each simulation.

Fig. 5. (left) KE transfer rate from zero-frequency to near-inertial waves due to horizontal shear and strain, i.e., $F_{h}\left(E_{K}\right)$ from Eqn. (15), (middle) KE transfer rate due to vertical shear, i.e., $F_{v}\left(E_{K}\right)$ from Eqn. (15), (right) horizontal average of $F_{h}\left(E_{K}\right)$ and $F_{v}\left(E_{K}\right)$ as a function of HAB. (a,b) $J=0.6$, (c,d) $J=1$, (e,f) $J=2$. All values computed over the last $4 t_{I}$.

Fig. 6. Normalized time-mean $(\omega=0)$ horizontal velocity $\bar{u} / U$ and buoyancy $\bar{b} / N^{2}$ : (a,b) $J=0.6$, (c,d) $J=1$, (e,f) $J=2$. All values computed over the last $4 t_{I}$ and averaged in $y$-direction.

Fig. 7. Real parts of normalized horizontal velocity $\tilde{u}_{f} / U$, buoyancy $\tilde{b}_{f} / N^{2}$, and vertical velocity $\tilde{w}_{f} / U$, CD-filtered at $\omega=f$ as defined in Eqn. (9): (a,b,c) $J=0.6$, (d,e,f) $J=1,(\mathrm{~g}, \mathrm{~h}, \mathrm{i}) J=2$. All values computed over the last $4 t_{I}$ and averaged in $y$-direction. Black dashed lines in the buoyancy plots (middle column) indicate the freely propagating inertial wave slope $\alpha_{2}$ defined in Eqn. (19).

Fig. 8. Same as Fig. 5, only for KE transfer rates from zero-frequency to super-inertial internal waves.

Fig. 9. (Left) APE transfer rates from zero-frequency to near-inertial waves due horizontal buoyancy fluxes, i.e., $F_{h}\left(E_{A}\right)$ from Eqn. (16), (middle) sum of APE transfer rate $F_{h}\left(E_{A}\right)$ and KE transfer rate from vertical shear, i.e., $F_{v}\left(E_{K}\right)$ from Eqn. (15), (right) horizontal average of $F_{v}\left(E_{K}\right), F_{h}\left(E_{A}\right)$, and $F_{h}\left(E_{A}\right)+F_{v}\left(E_{K}\right)$ as a function of HAB. The "effective" transfer rate $F_{h}\left(E_{A}\right)+F_{v}\left(E_{K}\right) \approx F_{v}^{e f f}$ (from Eqn. (18)) should be zero at $\omega=f$. (a,b) $J=0.6$, (c,d) $J=1$, (e,f) $J=2$. All values computed over the last $4 t_{I}$.

Fig. 10. Same as Fig. 9, only for APE and KE transfer rates from zero-frequency to super-inertial internal waves.

Fig. 11. Volume-averaged (a) mixing efficiency, $\eta_{V}$ defined in Eqn. (20), (b) APE and KE dissipation rates $\left(D\left(E_{A}\right)\right.$ marked with squares and $D\left(E_{K}\right)$ marked with stars, respectively), (c) internal 
energy transfer from APE to KE reservoir, $\overline{w^{\prime} b^{\prime}}$, and (d) APE transfer from near-inertial super-inertial internal waves, i.e., $F\left(E_{A}^{f}, E_{A}^{S I W}\right)$ as defined in Eqn. (22). All values are computed over four spatial intervals above the bottom: $H A B<0.1, H A B \in[0.1,0.2)$, $H A B \in[0.2,0.3)$, and $H A B \in[0.3,0.4)$. All values computed over the last $4 t_{I}$. Three different topographic regimes are presented: $J=0.6$ in blue, $J=1$ in red, and $J=2$ in black.

Fig. 12. Local mixing efficiency, $\eta$ defined in Eqn. (21), averaged in $y$ : (a) $J=0.6$, (b) $J=1$, and (c) $J=2$. All values computed over the last $4 t_{I}$.

Fig. 13. (a,b) Horizontally averaged APE dissipation rate $D\left(E_{A}\right),(\mathrm{c}, \mathrm{d}) \mathrm{KE}$ dissipation rate $D\left(E_{K}\right)$ : (left) dissipation rates of $\omega=0$ motions (solid lines) and internal waves (IW, dashed); (right) breakdown of internal wave dissipation rates into near-inertial (NIW, dash-dot) and superinertial (SIW, dotted) internal waves. All values computed over the last $4 t_{I}$. Three different topographic regimes are presented: $J=0.6$ in blue, $J=1$ in red, and $J=2$ in black. 


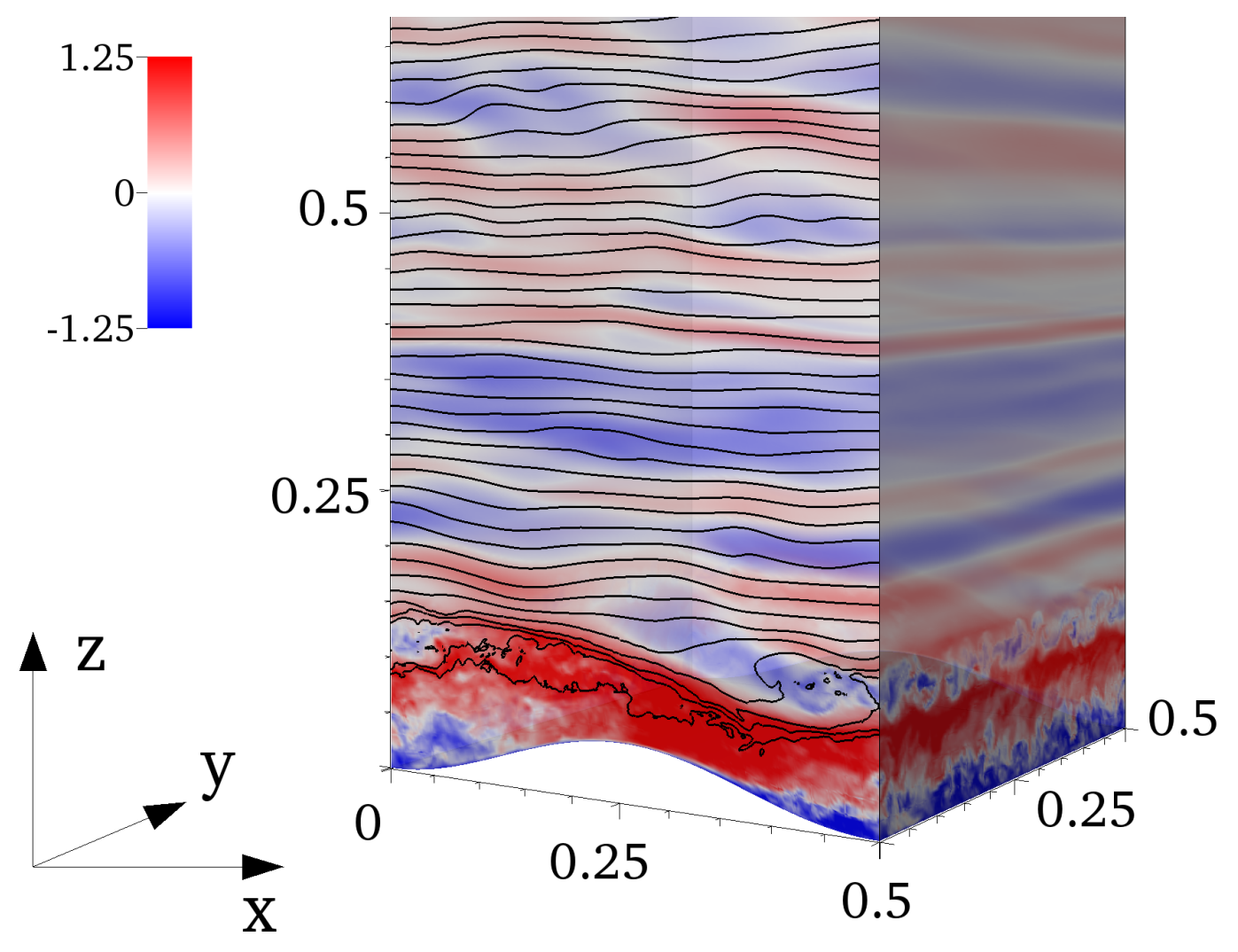

FIG. 1. Domain set-up for all simulations with sinusoidal topographic bump (upper part of the domain cropped).

Overlayed is a snapshot of the flow for experiment $(\chi, J)=(0.16,2)$ at $t=6.75 t_{I}$ to highlight a breaking event downstream of the topography.. Color: normalized perturbation velocity $u / U$; black contours: isopycnals.

Topography is homogeneous in $y$ and the domain is periodic in $x$ and $y$. 

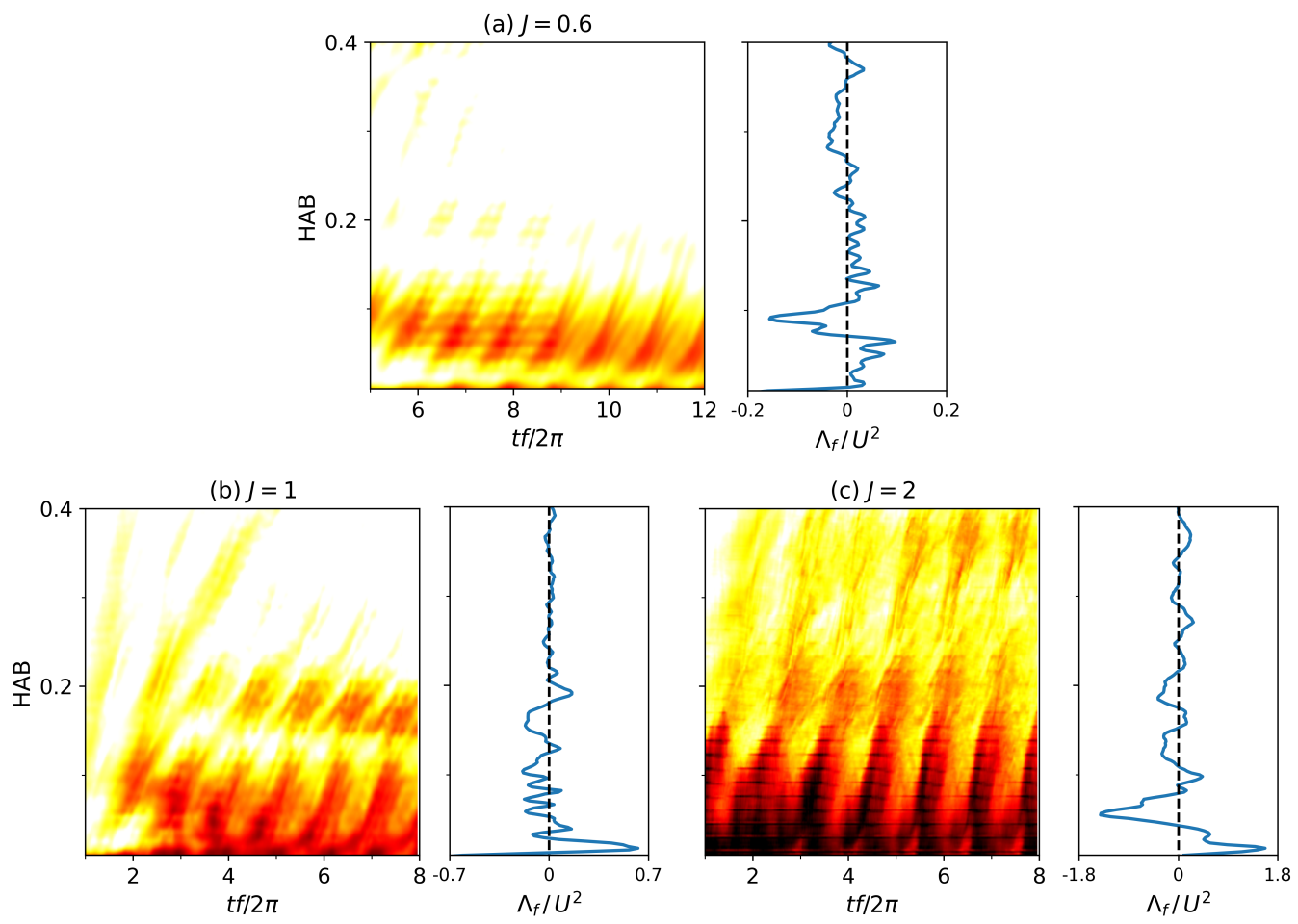

FIG. 2. (Left) Hovmöller diagram of the normalized total kinetic energy dissipation $\left(\log _{10}\left(E_{K} / U^{2}\right)\right)$ horizontally averaged and plotted in terms of height above the bottom (HAB) and (right) horizontally-averaged non-linear forcing for the near-inertial frequency motions, $\Lambda_{f}$ defined in Eqn. (10) computed over the last $4 t_{I}$.

(a) $J=0.6$, (b) $J=1$, and (c) $J=2$. 


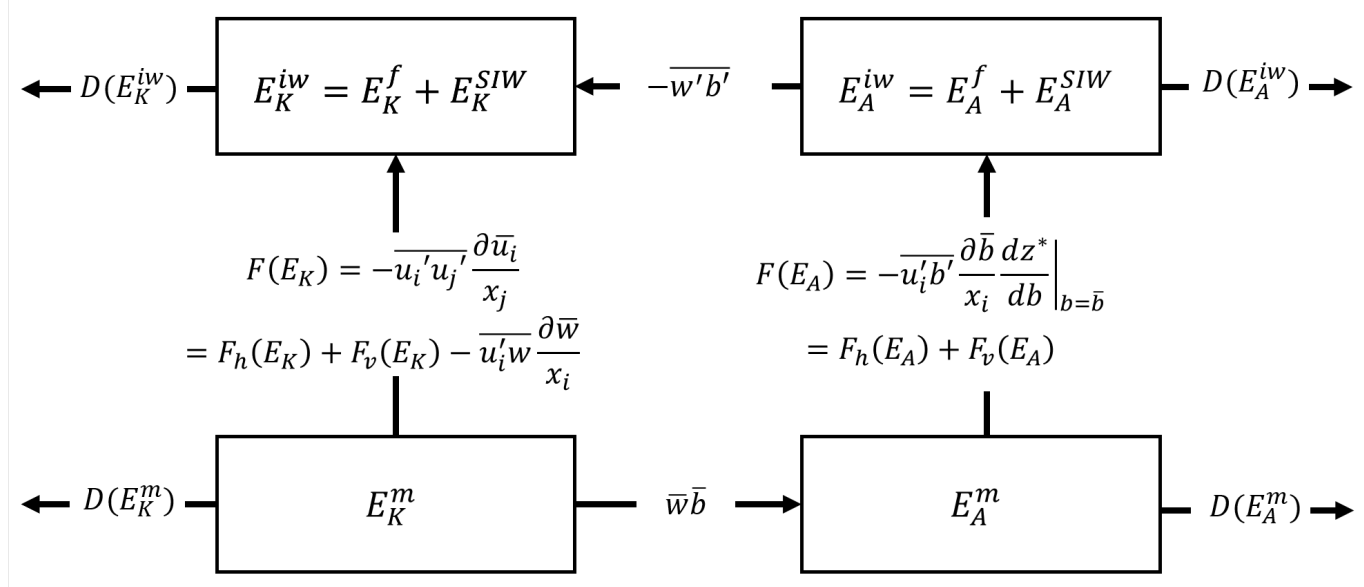

FIG. 3. Energy budget diagram illustrating KE and APE exchanges between the time-mean flow and internal waves, and dissipation rates of the time mean flow and internal waves. KE and APE are defined in Eqns. (7) and (8), respectively. KE and APE exchange rates are defined in Eqns. (15) and (16), respectively. The dissipation rates of KE and APE are defined in Eqns. (13) and (14), respectively. When a term on an arrow is positive (negative), energy goes along (opposite to) the direction of said arrow. 
(a) $E_{K}: \quad J=0.6$

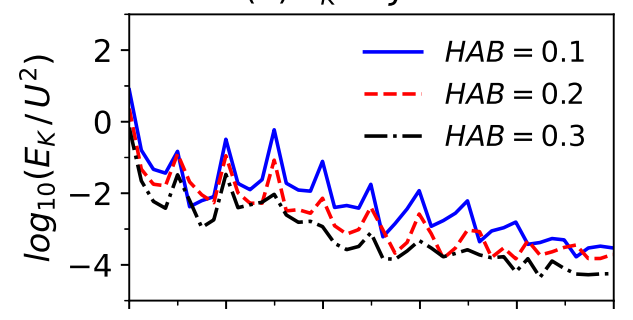

(c) $E_{K}: \quad J=1$

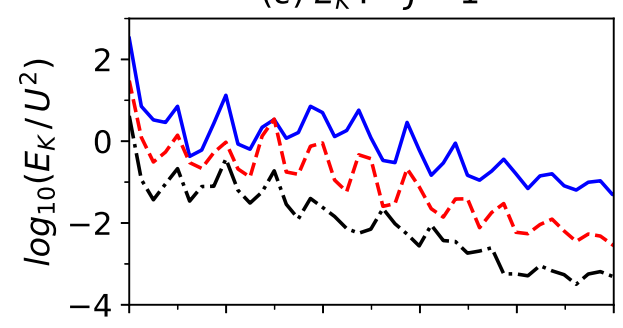

(e) $E_{K}: \quad J=2$

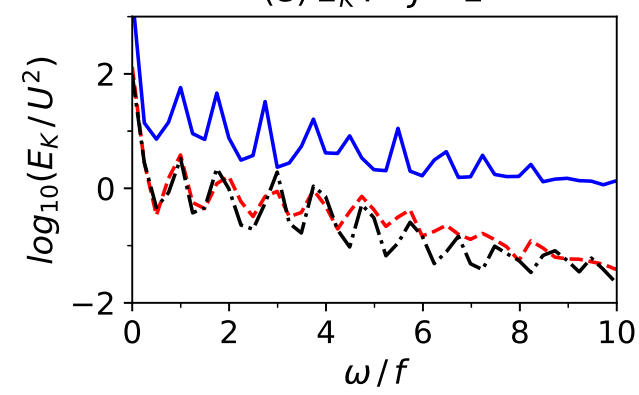

(b) $E_{A}: \quad J=0.6$

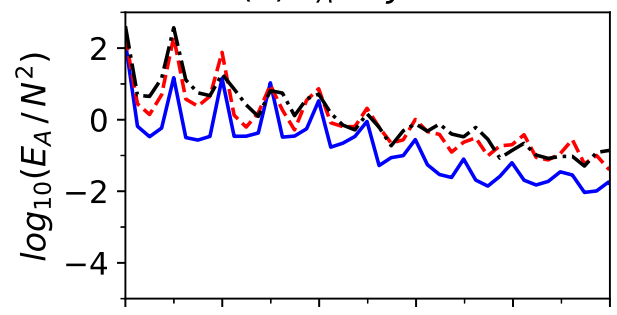

(d) $E_{A}: \quad J=1$

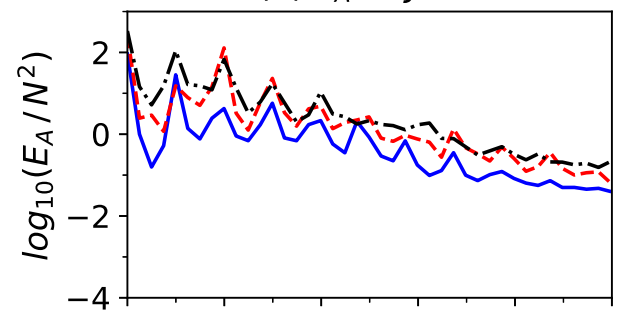

(f) $E_{A}: \quad J=2$

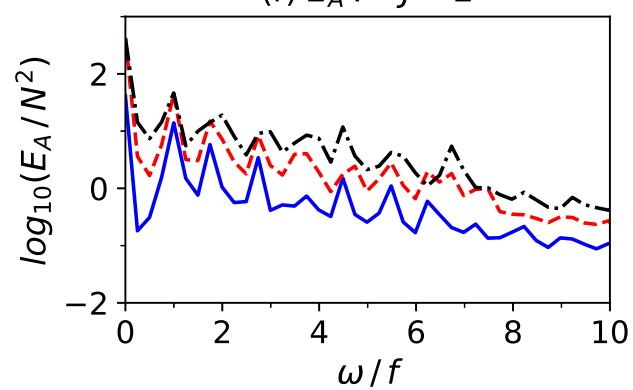

FIG. 4. (a,c) Normalized kinetic energy $\left(E_{K}\right)$ spectra, and (b,d) available potential energy $\left(E_{A}\right)$ spectra, excluding the background geostrophic flow, averaged at $H A B=0.1,0.2,0.3$. (Top) simulation with linear topography $J=0.6$, and (bottom) simulation with non-linear topography $J=2$. Spectra are computed over the last $4 t_{I}$ of each simulation. 

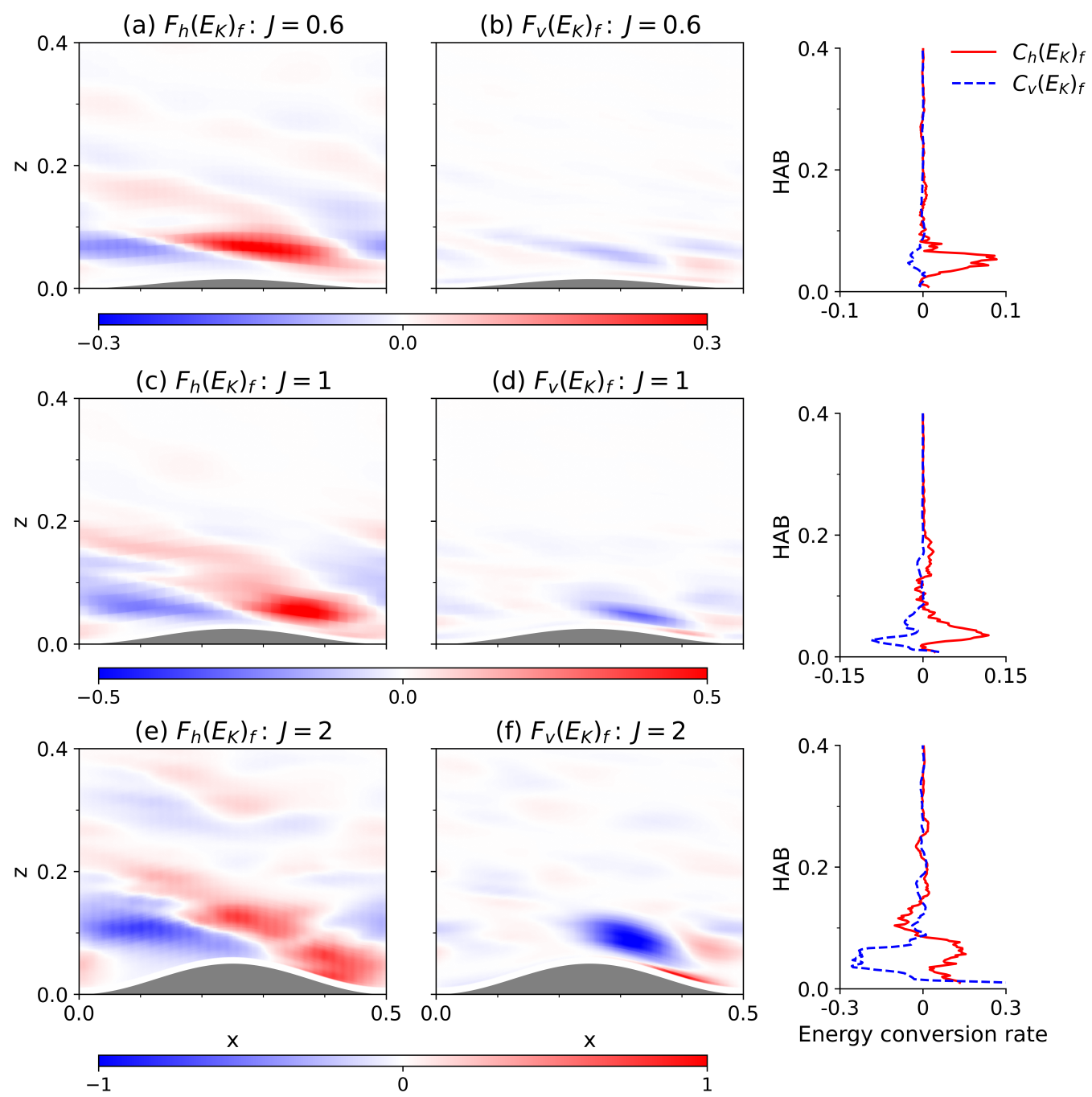

FIG. 5. (left) KE transfer rate from zero-frequency to near-inertial waves due to horizontal shear and strain, i.e., $F_{h}\left(E_{K}\right)$ from Eqn. (15), (middle) $\mathrm{KE}$ transfer rate due to vertical shear, i.e., $F_{v}\left(E_{K}\right)$ from Eqn. (15), (right) horizontal average of $F_{h}\left(E_{K}\right)$ and $F_{v}\left(E_{K}\right)$ as a function of HAB. (a,b) $J=0.6$, (c,d) $J=1$, (e,f) $J=2$. All values computed over the last $4 t_{I}$. 

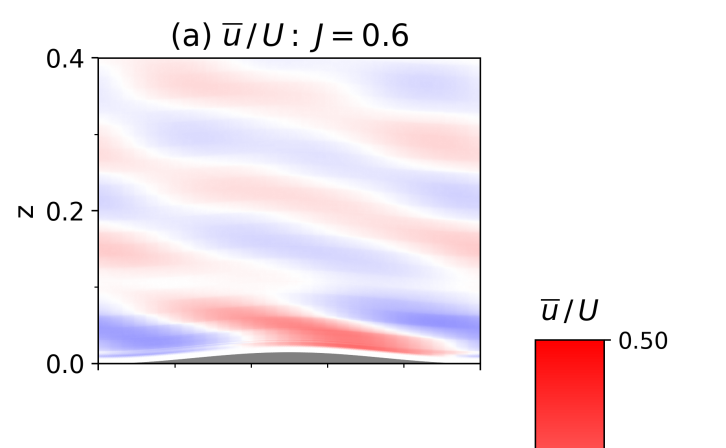

(b) $\bar{b} / N^{2}: J=0.6$
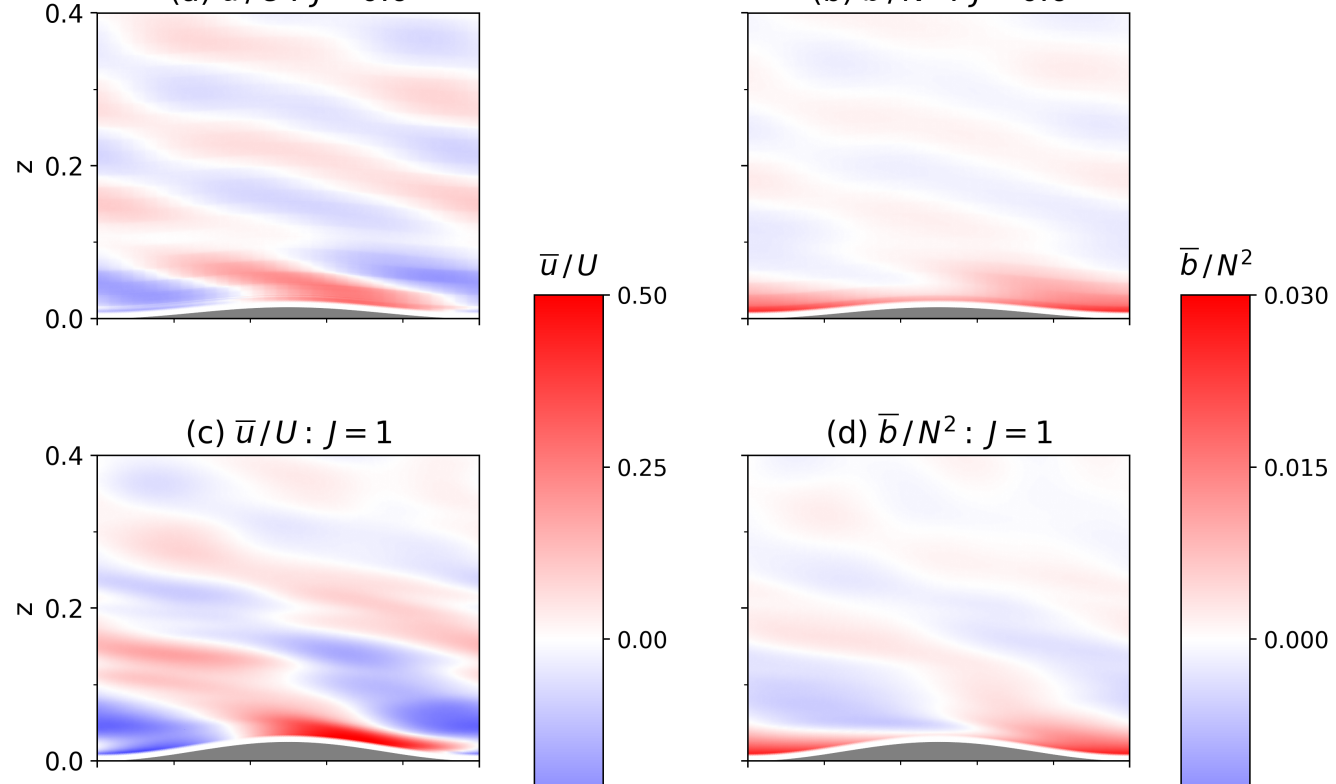

(d) $\bar{b} / N^{2}: J=1$

$-0.25$

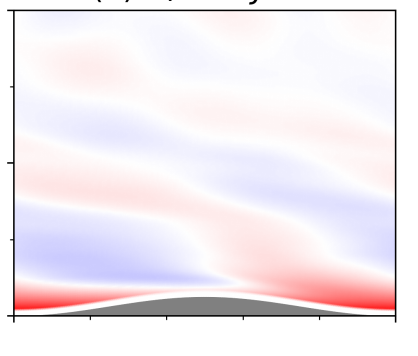

0.015
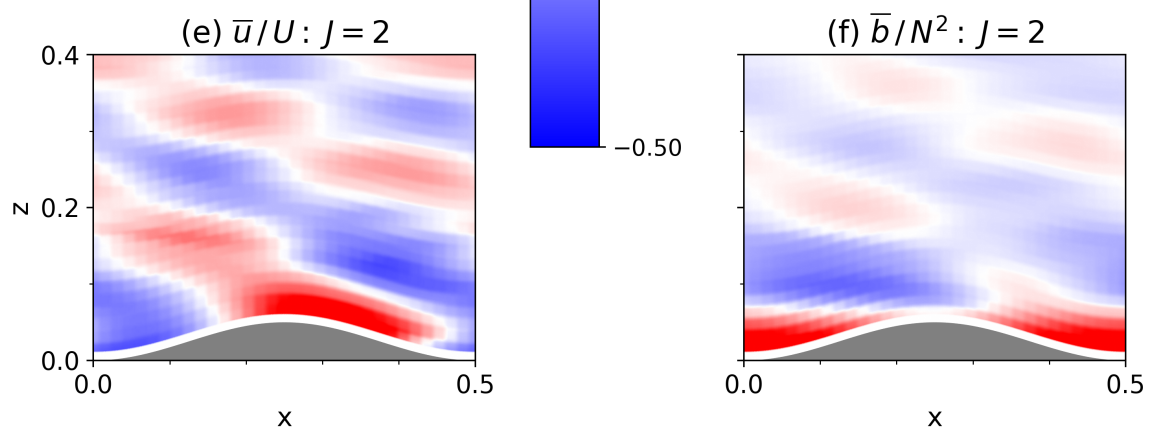

$-0.015$ $-0.25$
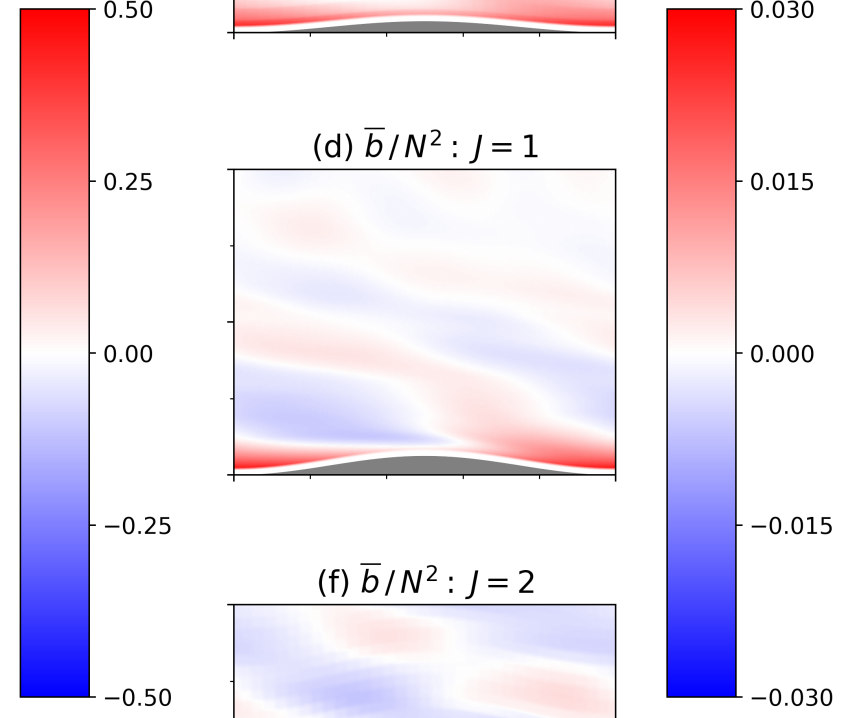

FIG. 6. Normalized time-mean $(\omega=0)$ horizontal velocity $\bar{u} / U$ and buoyancy $\bar{b} / N^{2}$ : (a,b) $J=0.6$, (c,d) $J=1$, (e,f) $J=2$. All values computed over the last $4 t_{I}$ and averaged in $y$-direction. 

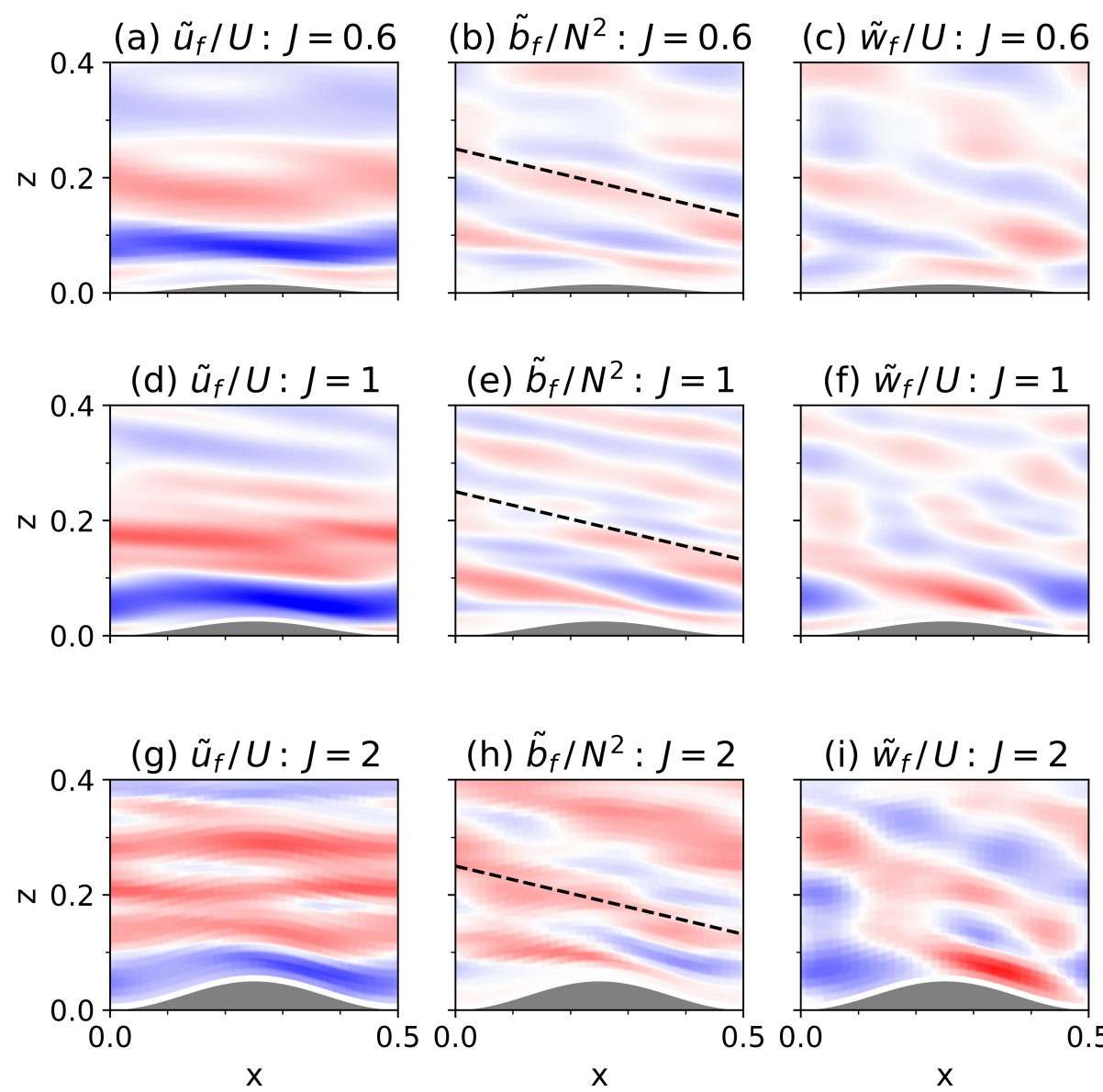

(h) $\tilde{b}_{f} / N^{2}: J=2$

(i) $\tilde{w}_{f} / U: J=2$
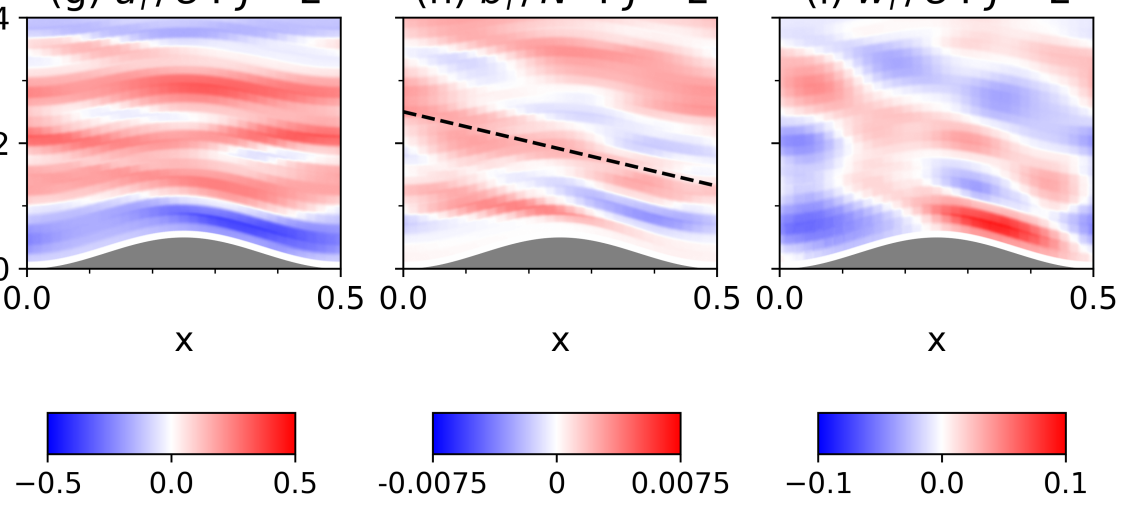

FIG. 7. Real parts of normalized horizontal velocity $\tilde{u}_{f} / U$, buoyancy $\tilde{b}_{f} / N^{2}$, and vertical velocity $\tilde{w}_{f} / U$, CD-filtered at $\omega=f$ as defined in Eqn. (9): (a,b,c) $J=0.6$, (d,e,f) $J=1,(\mathrm{~g}, \mathrm{~h}, \mathrm{i}) J=2$. All values computed over the last $4 t_{I}$ and averaged in $y$-direction. Black dashed lines in the buoyancy plots (middle column) indicate the freely propagating inertial wave slope $\alpha_{2}$ defined in Eqn. (19). 

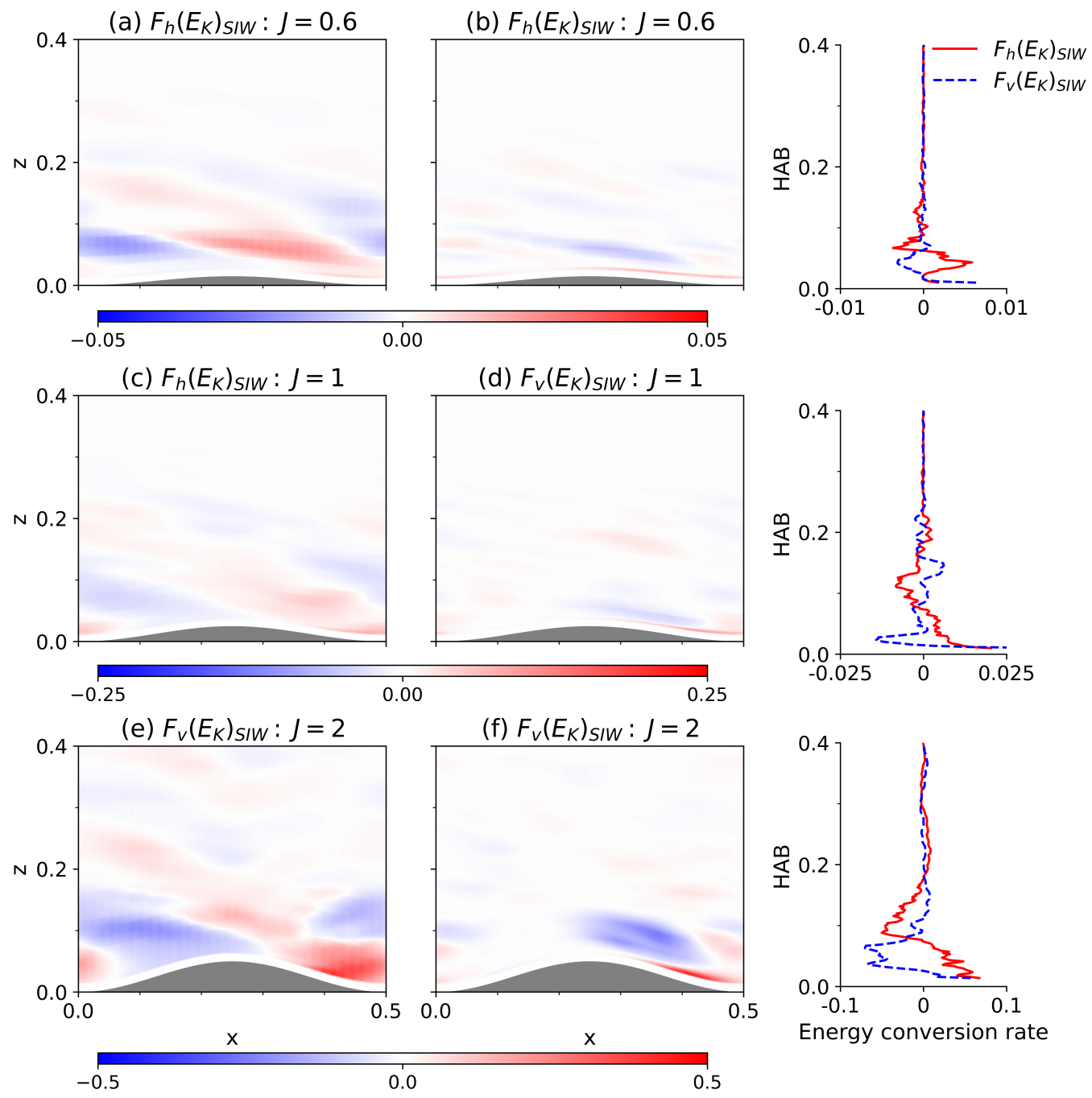

FIG. 8. Same as Fig. 5, only for KE transfer rates from zero-frequency to super-inertial internal waves. 

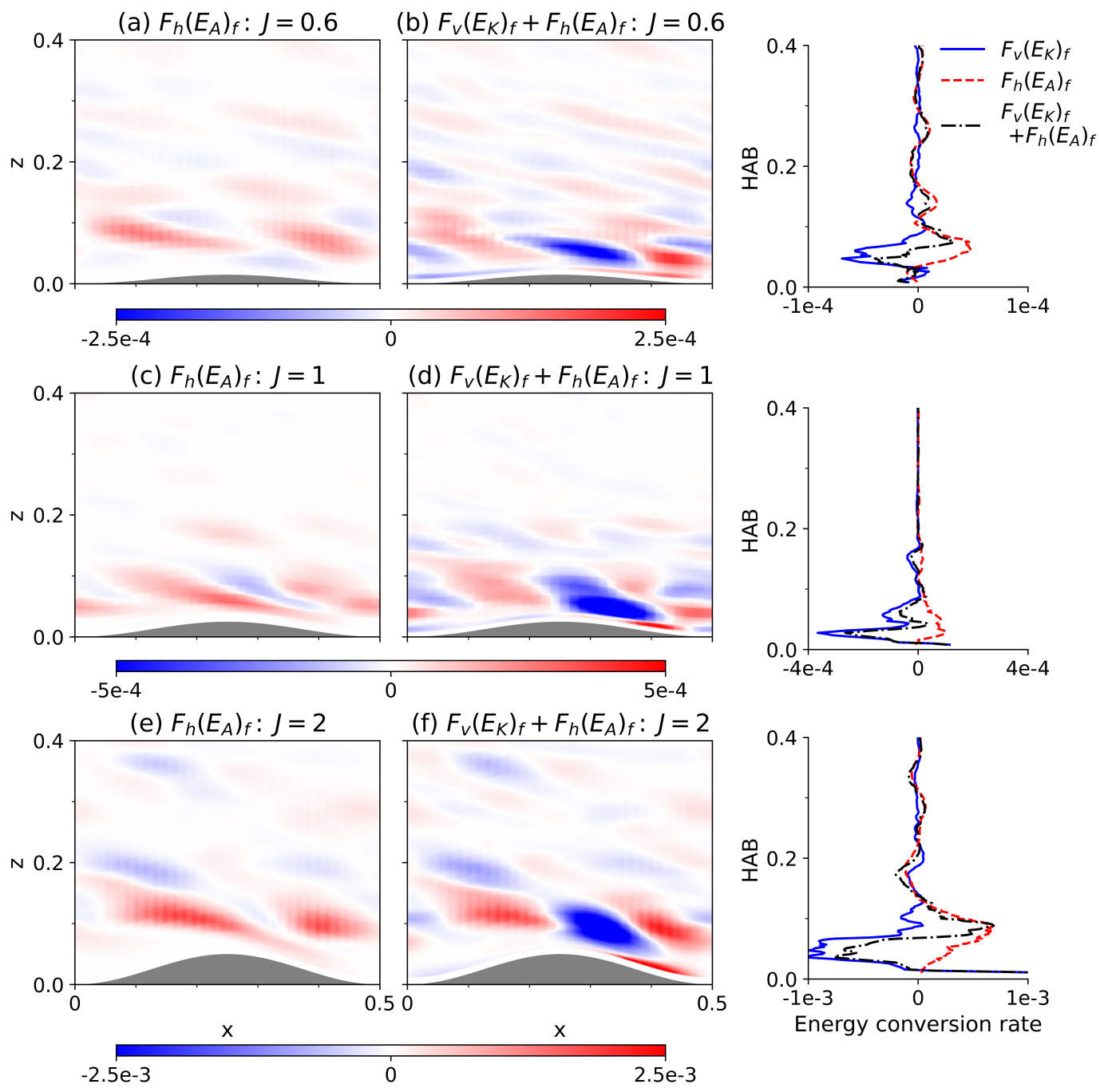

FIG. 9. (Left) APE transfer rates from zero-frequency to near-inertial waves due horizontal buoyancy fluxes, i.e., $F_{v}\left(E_{K}\right)$ from Eqn. (15), (right) horizontal average of $F_{v}\left(E_{K}\right), F_{h}\left(E_{A}\right)$, and $F_{h}\left(E_{A}\right)+F_{v}\left(E_{K}\right)$ as a function of HAB. The "effective" transfer rate $F_{h}\left(E_{A}\right)+F_{v}\left(E_{K}\right) \approx F_{v}^{e f f}$ (from Eqn. (18)) should be zero at $\omega=f$. (a,b) $J=0.6$, (c,d) $J=1$, (e,f) $J=2$. All values computed over the last $4 t_{I}$. 

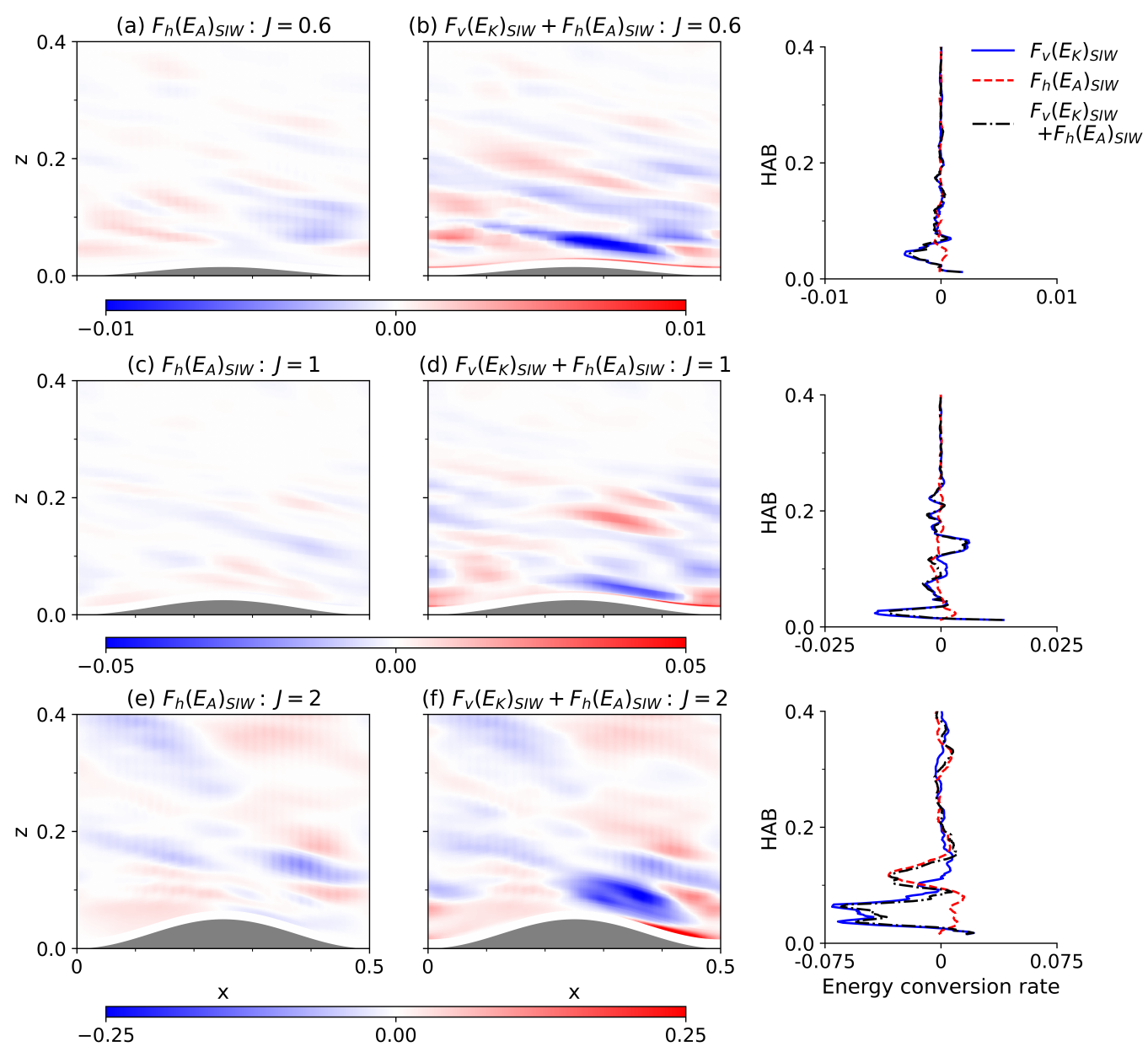

FIG. 10. Same as Fig. 9, only for APE and KE transfer rates from zero-frequency to super-inertial internal waves. 
(a)

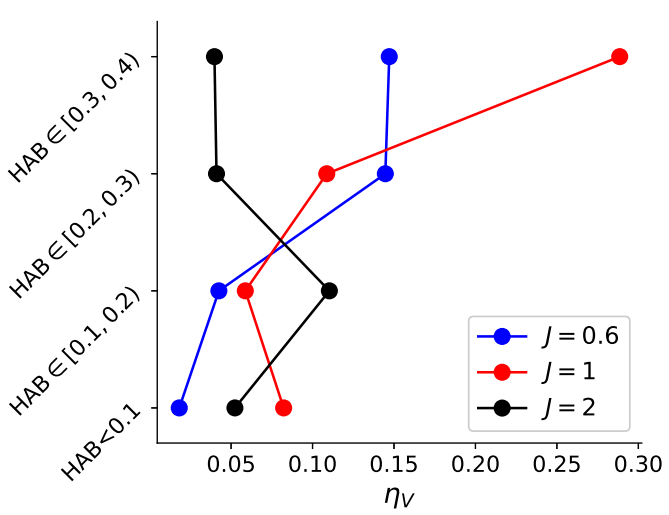

(c)

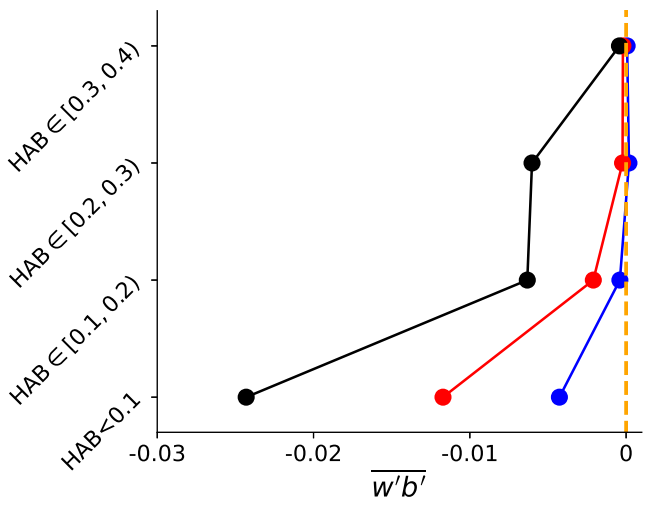

(b)

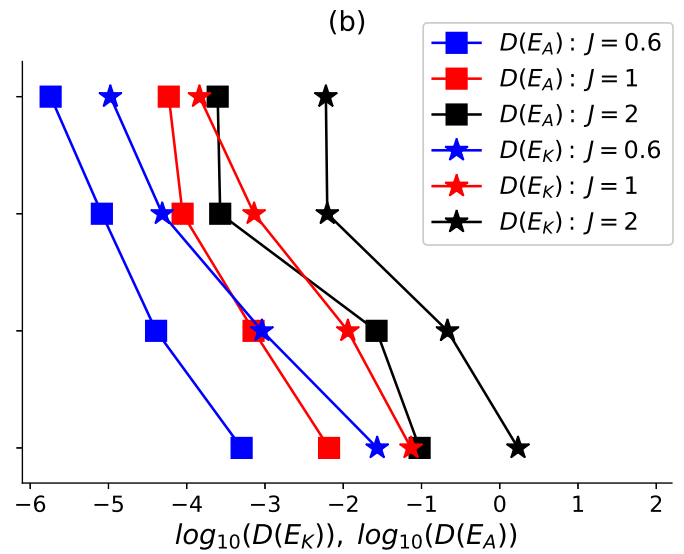

(d)

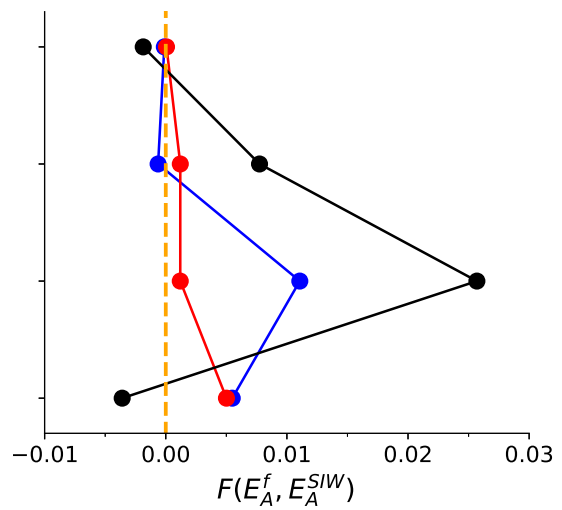

FIG. 11. Volume-averaged (a) mixing efficiency, $\eta_{V}$ defined in Eqn. (20), (b) APE and KE dissipation rates ( $D\left(E_{A}\right)$ marked with squares and $D\left(E_{K}\right)$ marked with stars, respectively), (c) internal energy transfer from APE to KE reservoir, $\overline{w^{\prime} b^{\prime}}$, and (d) APE transfer from near-inertial super-inertial internal waves, i.e., $F\left(E_{A}^{f}, E_{A}^{S I W}\right)$ as defined in Eqn. (22). All values are computed over four spatial intervals above the bottom: $H A B<0.1$, $H A B \in[0.1,0.2), H A B \in[0.2,0.3)$, and $H A B \in[0.3,0.4)$. All values computed over the last $4 t_{I}$. Three different topographic regimes are presented: $J=0.6$ in blue, $J=1$ in red, and $J=2$ in black. 


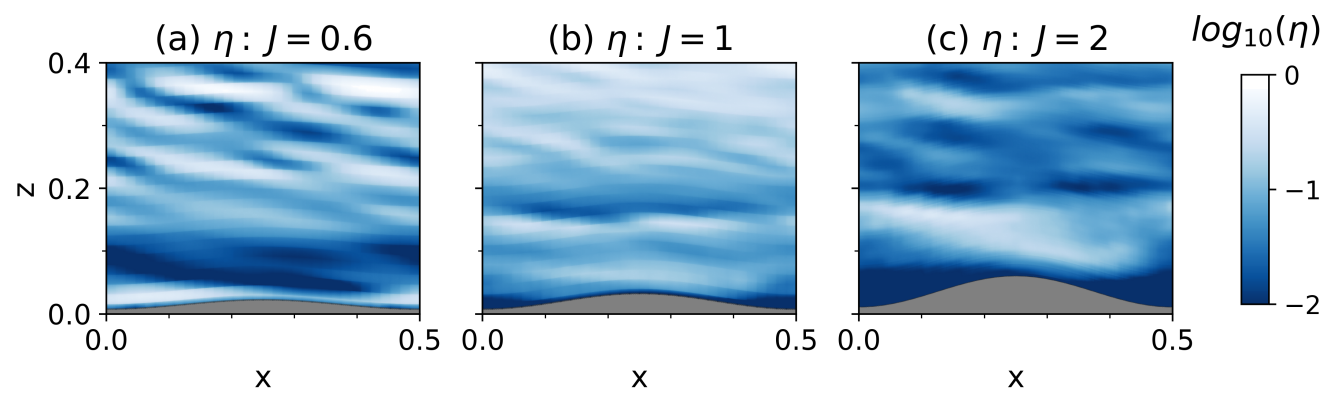

FIG. 12. Local mixing efficiency, $\eta$ defined in Eqn. (21), averaged in $y$ : (a) $J=0.6$, (b) $J=1$, and (c) $J=2$.

All values computed over the last $4 t_{I}$. 
(a)

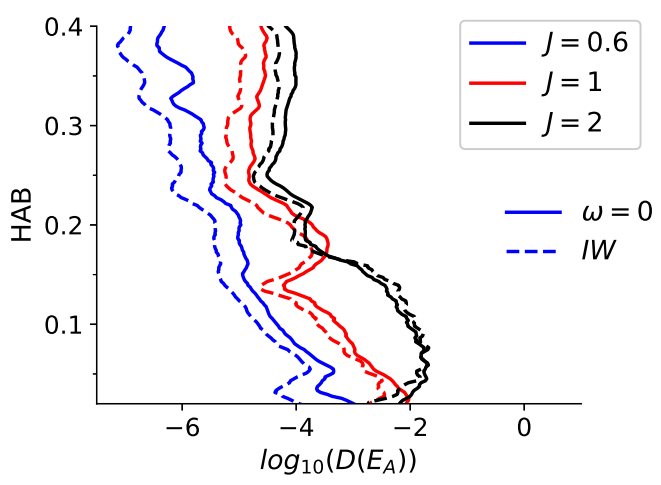

(c)

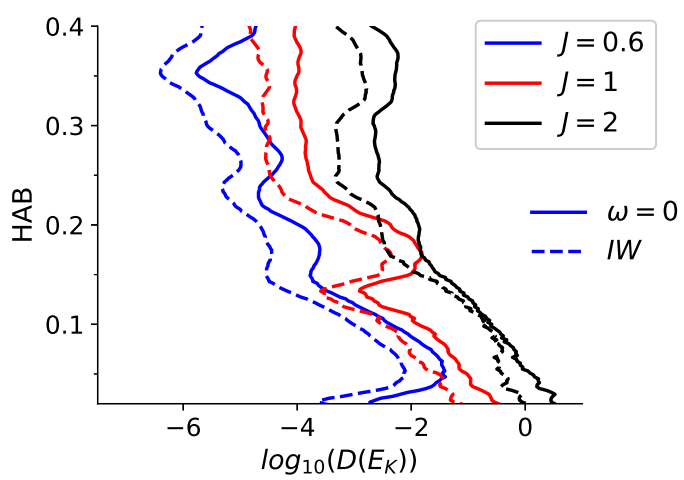

(b)

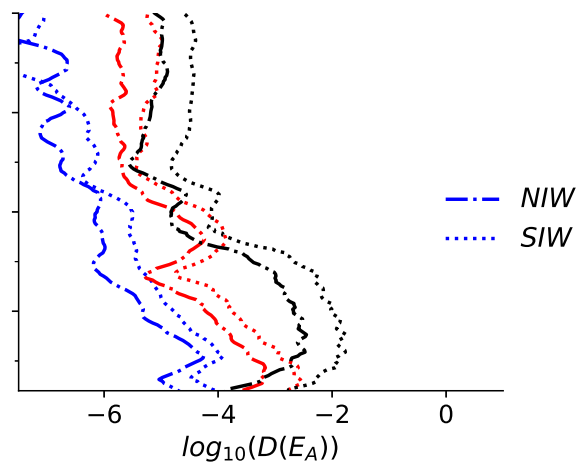

(d)

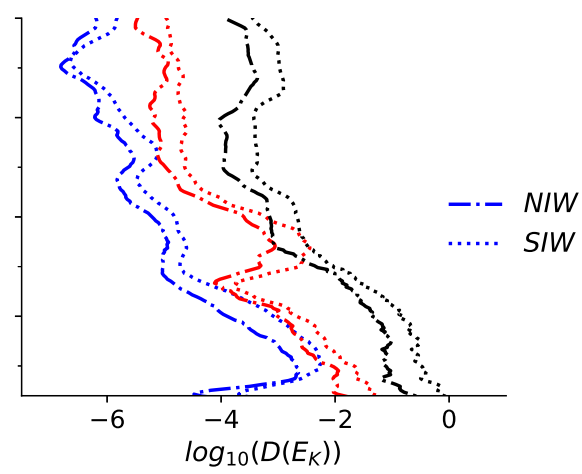

FIG. 13. (a,b) Horizontally averaged APE dissipation rate $D\left(E_{A}\right),(\mathrm{c}, \mathrm{d}) \mathrm{KE}$ dissipation rate $D\left(E_{K}\right)$ : (left) dissipation rates of $\omega=0$ motions (solid lines) and internal waves (IW, dashed); (right) breakdown of internal wave dissipation rates into near-inertial (NIW, dash-dot) and super-inertial (SIW, dotted) internal waves. All values computed over the last $4 t_{I}$. Three different topographic regimes are presented: $J=0.6$ in blue, $J=1$ in red, and $J=2$ in black. 